\title{
Weighted piecewise pseudo double-almost periodic solution for impulsive evolution equations
}

\author{
Chao Wang ${ }^{a, b, *}$, Ravi P. Agarwal ${ }^{b}$, Donal O’Regan ${ }^{c}$ \\ ${ }^{a}$ Department of Mathematics, Yunnan University, Kunming, Yunnan 650091, People's Republic of China. \\ ${ }^{b}$ Department of Mathematics, Texas A\&M University-Kingsville, 700 University Blvd., TX 78363-8202, Kingsville, USA. \\ 'School of Mathematics, Statistics and Applied Mathematics, National University of Ireland, Galway, Ireland. \\ Communicated by M. Bohner
}

\begin{abstract}
In this paper, based on the concept and properties of almost-complete closedness time scales (ACCTS), we investigate the existence of weighted pseudo double-almost periodic mild solutions for non-autonomous impulsive evolution equations. We also consider the exponential stability of weighted pseudo double-almost periodic solutions. Finally, we conclude our paper by providing several illustrative applications to different types of dynamic equations and mathematical models. These applications justify the practical usefulness of the established theoretical results. (C)2017 All rights reserved.
\end{abstract}

Keywords: Time scales, weighted pseudo double-almost periodic solution, impulsive evolution equations. 2010 MSC: 34N05, 43A60, 34A37, 34C27.

\section{Introduction}

The theory of time scales was introduced by Hilger in his Ph.D. thesis in 1988 [17] to unify continuous and discrete analysis. "Dynamic equations on time scales" contains, links and extends the classical theory of differential $(\mathbb{T}=\mathbb{R})$ and difference $(\mathbb{T}=\mathbb{Z})$ equations. The theory of dynamic equations on time scales also extends to cases "in between", e.g., to the so-called q-difference equations $\left(\mathbb{T}=q^{\mathbb{N}_{0}}:=\left\{q^{t}\right.\right.$ : $\mathrm{t} \in \mathbb{N}_{0}$ for $\left.\left.\mathrm{q}>1\right\}\right)$ or $\left(\mathbb{T}=\overline{\mathrm{q}^{\mathbb{Z}}}:=\mathrm{q}^{\mathbb{Z}} \cup\{0\}\right)$, and can be applied on different types of time scales such as $\mathbb{T}=h \mathbb{N}, \mathbb{T}=\mathbb{N}^{2}$, and $\mathbb{T}=\mathbb{T}_{n}$ the space of the harmonic numbers. Several authors have expounded on various aspects of this new theory (see $[1,2,4,7,18,33]$ ).

The theory of almost periodic functions and systems initiated by Bohr was largely developed in the last century (see $[5,14]$ ), and many mathematicians consider almost periodic problems that exist in the change and development in nature (see $[8,9])$. To find a class of functions which are more general than almost periodic functions, the notion of pseudo-almost periodic functions was introduced by Zhang in $[35,36]$. Generalizations were considered in [11, 13, 21] and the references therein. In [12], Diagana

\footnotetext{
*Corresponding author

Email addresses: chaowang@ynu. edu.cn (Chao Wang), Agarwal@tamuk. edu (Ravi P. Agarwal), donal. oregan@nuigalway . ie (Donal O'Regan)
}

doi:10.22436/jnsa.010.07.41

Received 2017-01-04 
introduced a new concept which he called weighted pseudo-almost periodicity. The concepts of almost periodicity and pseudo-almost periodicity, and their applications to dynamic equations on time scales are very recent $[3,15,27,29]$. However, all discussions of these topics are restricted to periodic time scales and there is little work on almost periodic time scales. From a practical point of view, although we live in the real world in which almost all natural phenomena move in circles, there still exist many natural phenomena which cannot be described by periodic time scales. The required time intervals for the recurrence of many common natural phenomena are usually not periodic. For example, the required time intervals of a round for a celestial body motion, sun or earth etc., are "almost equivalent" but are not precisely the same. If we consider the revolution of the earth, then we see that the position of the earth is almost overlapping after almost a year rather than after a year. The second "almost" for the time should not be ignored. Another example is tidal flood where its required time intervals of recurrence can also not be precisely described by periodic time scales because every time interval is "almost equivalent". Hence, it is more realistic to consider that the object's status with periodicity or almost periodicity will be always the same or almost the same after "an almost-equivalent time interval". In such a situation, we say the object is with "double almost periodicity" (that is, the status and its time variable of the object are almost periodic simultaneously). Time scales $h \mathbb{Z}$ and $\mathbb{R}$ have some very nice properties so that the dynamic change of functions established on them can be described well because of the closedness for the translation of time variables. All periodic time scales have a nice closedness for the translation of time variables (see [20,33]), and we say $\mathbb{T}$ is with "complete closedness" (CCTS). A time scale with such a type of "complete closedness" assists in conquering the difficulties of defining and studying functions on time scales. However, there exists a type of time scales that has "almost-complete closedness" but not "complete closedness" (see [28, 30]). This type of time scales can describe the "double almost periodicity" of an object's status with almost periodic dynamic behavior in the real world and it is more general and comprehensive than periodic time scales. Therefore, introducing and studying functions on time scales with "almost-complete closedness" is important.

In addition, many phenomena in nature are characterized by the fact that their states are subject to sudden changes at certain moments and can be described by impulsive systems (see [23, 31]). The existence of almost periodic solutions for abstract impulsive dynamic equations was considered by many authors; see [10, 16, 24, 25]. To the best of our knowledge, weighted piecewise pseudo double-almost periodic solutions for impulsive dynamic equations on ACCTS have never been considered in any literature.

To fill this gap in the literature, in this paper, we investigate the existence and uniqueness of weighted pseudo double-almost periodic mild solutions for impulsive evolution equations. Specifically, we study the $\varepsilon$-equivalent impulsive evolution equations ( $\varepsilon$-EIEE, see Definition 2.13) on time scales given by

$$
\left\{\begin{array}{l}
x^{\Delta}(t)=A(t) x(t)+f(t, x), t \in \mathscr{R}_{\mathbb{T}}(\tau, \varepsilon), t \neq t_{k_{i}, \varepsilon}, i \in \mathbb{Z}, \\
\Delta x\left(t_{k_{i}, \varepsilon}\right)=x\left(t_{k_{i}, \varepsilon}^{+}\right)-x\left(t_{k_{i}, \varepsilon}^{-}\right)=I_{k}\left(x\left(t_{k_{i}, \varepsilon}\right)\right), t=t_{k_{i}, \varepsilon} \in \cup \mathfrak{B}^{\varepsilon}, i \in \mathbb{Z},
\end{array}\right.
$$

where $A \in \mathrm{PC}_{\mathrm{rd}}(\mathbb{T}, \mathrm{B}(\mathbb{X}))$ is a linear operator on the Banach space $\mathbb{X}$ and $f \in \mathrm{PC}_{\mathrm{rd}}(\mathbb{T} \times \mathbb{X}, \mathbb{X})$. Now $f, I_{i}, t_{i}$ satisfy suitable conditions that will be given later, and $\mathbb{T}$ is an almost-complete closedness time scale (see Definition 2.6, $\mathscr{R}_{\mathbb{T}}(\tau, \varepsilon):=\mathbb{T} \cap\left(\cup_{\tau \in \Pi_{\varepsilon}} \overline{\mathbb{T}^{-\tau} \backslash A_{-\tau}^{\varepsilon}}\right), \Pi_{\varepsilon}=E\{\mathbb{T}, \varepsilon\}, A_{\tau}^{\varepsilon}$ is an $\varepsilon$-improper set of $\mathbb{T}$, where $E\{\mathbb{T}, \varepsilon\}$ is an $\varepsilon$-translation number set of $\mathbb{T}$ ) and

$$
\mathfrak{B}^{\varepsilon}=\left\{\left\{t_{k_{i}, \varepsilon}\right\} \subset\left\{t_{k}\right\} \subset \mathbb{T}: t_{k_{i}, \varepsilon} \in \mathscr{R}_{\mathbb{T}}(\tau, \varepsilon), t_{k_{i}, \varepsilon}<t_{k_{i+1}, \varepsilon}, i \in \mathbb{Z}, \lim _{i \rightarrow \infty} t_{k_{i}, \varepsilon}=\infty\right\},
$$

which denotes all unbounded increasing sequences of real numbers and

$$
\cup \mathfrak{B}^{\varepsilon}=\left\{t_{k_{i}, \varepsilon} \in\left\{t_{k}\right\}: t_{k_{i}, \varepsilon} \in \mathscr{R}_{\mathbb{T}}(\tau, \varepsilon), t_{k_{i}, \varepsilon}<t_{k_{i+1}, \varepsilon}, i \in \mathbb{Z}, \lim _{i \rightarrow \infty} t_{k_{i}, \varepsilon}=\infty\right\} .
$$

The notations $x\left(t_{k_{i}, \varepsilon}^{+}\right)$and $x\left(t_{k_{i}, \varepsilon}^{-}\right)$represent the right-hand and the left-hand side limits of $x(\cdot)$ at $t_{k_{i}, \varepsilon}$, respectively. 
The organization of this paper is as follows. In Section 2, we collect some preliminary results concerning the theory of time scales and introduce the concept of $\varepsilon$-equivalent impulsive evolution equations. In Section 3, conditions on the existence and uniqueness of weighted pseudo double-almost periodic (mild) solutions for impulsive evolution equations on ACCTS are obtained. Also the exponential stability of weighted pseudo double-almost periodic solutions is considered. In Sections 4, several illustrative applications are provided which include applications to IBVP for $\Delta$-partial dynamic equations and mathematical models.

\section{2. $\varepsilon$-equivalent impulsive evolution equations}

In this section, we first recall some basic definitions and lemmas which will be used in this paper. For more details of time scales, see [7].

Let $\mathbb{T}$ be a nonempty closed subset (time scale) of $\mathbb{R}$. The forward and backward jump operators $\sigma, \rho: \mathbb{T} \rightarrow \mathbb{T}$ and the graininess $\mu: \mathbb{T} \rightarrow \mathbb{R}^{+}$are defined, respectively, by

$$
\sigma(t)=\inf \{s \in \mathbb{T}: s>t\}, \quad \rho(t)=\sup \{s \in \mathbb{T}: s<t\}, \quad \mu(t)=\sigma(t)-t .
$$

Definition 2.1 ([7]). The function $f: \mathbb{T} \rightarrow \mathbb{X}$ is called rd-continuous provided that it is continuous at each right-dense point and has a left-sided limit at left dense points, and we write $f \in C_{r d}(\mathbb{T})=C_{r d}(\mathbb{T}, \mathbb{X})$. Let $t \in \mathbb{T}^{k}$, and the Delta derivative of $f$ at $t$ denoted as $f^{\Delta}(t)$ satisfies the inequality

$$
\left|f(\sigma(t))-f(s)-f^{\Delta}(t)[\sigma(t)-s]\right| \leqslant \varepsilon|\sigma(t)-s|
$$

for any $\varepsilon>0$ and all $s \in U$; here $U$ is a neighborhood of $t$. Let $F \in C(\mathbb{T}, \mathbb{X})$ be a function, and it is called antiderivative of $f: \mathbb{T} \rightarrow \mathbb{X}$ provided $F^{\Delta}(t)=f(t)$ for each $t \in \mathbb{T}^{k}$. If $F^{\Delta}(t)=f(t)$, then we define the Delta integral by $\int_{a}^{t} f(s) \Delta s=F(t)-F(a)$.

Definition 2.2 ([7]). A function $p: \mathbb{T} \rightarrow \mathbb{R}$ is called $\mu$-regressive provided $1+\mu(t) p(t) \neq 0$ for all $t \in \mathbb{T}^{k}$. The set of all regressive and rd-continuous functions $p: \mathbb{T} \rightarrow \mathbb{R}$ will be denoted by $\mathcal{R}=\mathcal{R}(\mathbb{T})=\mathcal{R}(\mathbb{T}, \mathbb{R})$. We define the set $\mathcal{R}^{+}=\mathcal{R}^{+}(\mathbb{T}, \mathbb{R})=\{p \in \mathcal{R}: 1+\mu(t) p(t)>0, \forall t \in \mathbb{T}\}$.

Definition 2.3 ([7]). If $r$ is a regressive function, then the generalized exponential function $e_{r}$ is defined by $e_{r}(t, s)=\exp \left\{\int_{s}^{t} \xi_{\mu(\tau)}(r(\tau)) \Delta \tau\right\}$ for all $s, t \in \mathbb{T}$, where the $\mu$-cylinder transformation is as in $\xi_{h}(z):=$ $\frac{1}{h} \log (1+z h)$.

Next, we introduce the concept of almost-complete closedness time scales which is more general than the concept of almost periodic time scales proposed in [28, 30, 31].

Let $\tau$ be a number and $A_{\tau}^{\varepsilon}$ be a subset of $\mathbb{R}, \bar{A}$ denotes the closure of the set $A$, and we set the time scales:

$$
\mathbb{T}:=\cup_{i \in I}\left[\alpha_{i}, \beta_{i}\right], \quad \mathbb{T}^{\tau}:=\mathbb{T}+\tau=\{t+\tau: \forall t \in \mathbb{T}\}:=\cup_{i \in I}\left[\alpha_{i}^{\tau}, \beta_{i}^{\tau}\right], \cup_{i \in I}\left[\tilde{\alpha}_{i}^{\tau}, \tilde{\beta}_{i}^{\tau}\right]=\overline{\mathbb{T} \backslash A_{\tau}^{\varepsilon}},
$$

and define the distance between two time scales $\overline{\mathbb{T} \backslash A_{\mathcal{\tau}}^{\varepsilon}}$ and $\mathbb{T}^{\tau}$ by

$$
\mathrm{d}\left(\overline{\mathbb{T} \backslash A_{\tau}^{\varepsilon}}, \mathbb{T}^{\tau}\right)=\max \left\{\sup _{i \in \mathrm{I}}\left|\tilde{\alpha}_{i}^{\tau}-\alpha_{i}^{\tau}\right|, \sup _{i \in \mathrm{I}}\left|\tilde{\beta}_{i}^{\tau}-\beta_{i}^{\tau}\right|\right\},
$$

where I is an infinite index set and

$$
\begin{array}{lll}
\alpha_{i}^{\tau}:=\inf \left\{\alpha \in \mathbb{T}^{\tau}:\left|\alpha_{i}-\alpha\right|\right\} & \text { and } & \beta_{i}^{\tau}:=\inf \left\{\beta \in \mathbb{T}^{\tau}:\left|\beta_{i}-\beta\right|\right\}, \\
\tilde{\alpha}_{i}^{\tau}:=\inf \left\{\alpha \in \overline{\mathbb{T} \backslash A_{\tau}^{\varepsilon}}:\left|\alpha_{i}^{\tau}-\alpha\right|\right\} & \text { and } & \tilde{\beta}_{i}^{\tau}:=\inf \left\{\beta \in \overline{\mathbb{T} \backslash A_{\tau}^{\varepsilon}}:\left|\beta_{i}^{\tau}-\beta\right|\right\} .
\end{array}
$$

Inspired by the relatively dense set in the book by Fink (see [14]), we introduce the following concept: 
Definition 2.4 ([29]). A subset $S$ of $\Pi$ is called relatively dense if there exists a positive number $L \in \Pi$ such that $[a, a+L]_{\Pi} \cap S \neq \emptyset$ for all $a \in \Pi$. The number $L$ is called the inclusion length.

In fact, Definition 2.4 can be generalized to the following definition of relatively dense set on any given subset of $\mathbb{R}$.

Definition 2.5. Let $\Pi_{\mathbb{R}}$ be an arbitrary subset of $\mathbb{R}$ and $A \subset \Pi_{\mathbb{R}}$. We say that $A$ is relatively dense in $\Pi_{\mathbb{R}}$ if there exists a positive number $l \in \Pi_{\mathbb{R}}$ such that for all $a \in \Pi_{\mathbb{R}}$ we have $[a, a+l]_{\Pi_{\mathbb{R}}} \cap A \neq \emptyset, l$ is called the inclusion length.

In what follows, we will give three equivalent definitions of ACCTS.

Let $\Pi:=\left\{\tau \in \mathbb{R}: \mathbb{T}_{\tau} \neq \emptyset\right\} \neq\{0\}$, where $\mathbb{T}_{\tau}=\mathbb{T} \cap \mathbb{T}^{\tau}$.

Definition 2.6. We say $\mathbb{T}$ is an almost-complete closedness time scale (ACCTS) if for any given $\varepsilon_{1}>0$, there exist a constant $l\left(\varepsilon_{1}\right)>0$ such that each interval of length $l\left(\varepsilon_{1}\right)$ contains a $\tau\left(\varepsilon_{1}\right)$ and sets $A_{\tau}^{\varepsilon_{1}}$ such that

$$
\mathrm{d}\left(\overline{\mathbb{T} \backslash A_{\tau}^{\varepsilon_{1}}}, \mathbb{T}^{\tau}\right)<\varepsilon_{1}
$$

i.e., for any $\varepsilon_{1}>0$, the following set

$$
\mathrm{E}\left\{\mathbb{T}, \varepsilon_{1}\right\}=\left\{\tau \in \Pi: \mathrm{d}\left(\overline{\mathbb{T} \backslash A_{\tau}^{\varepsilon_{1}}}, \mathbb{T}^{\tau}\right)<\varepsilon_{1}\right\}:=\Pi_{\varepsilon_{1}}
$$

is relatively dense in $\Pi$. Here, $\tau$ is called the $\varepsilon_{1}$-translation number of $\mathbb{T}, l\left(\varepsilon_{1}\right)$ is called the inclusion length of $\mathrm{E}\left\{\mathbb{T}, \varepsilon_{1}\right\}$, and $\mathrm{E}\left\{\mathbb{T}, \varepsilon_{1}\right\}$ the $\varepsilon_{1}$-translation set of $\mathbb{T}, A_{\tau}^{\varepsilon_{1}}$ is called the $\varepsilon_{1}$-improper set of $\mathbb{T}, \mathscr{R}_{\mathbb{T}}\left(\tau, \varepsilon_{1}\right):=$ $\mathbb{T} \cap\left(\cup_{\tau \in \Pi_{\varepsilon_{1}}} \overline{\mathbb{T}^{-\tau} \backslash A_{-\tau}^{\varepsilon_{1}}}\right)$ the $\varepsilon_{1}$-main region of $\mathbb{T}$, where $A_{-\tau}^{\varepsilon_{1}}=\left(A_{\tau}^{\varepsilon_{1}}\right)^{-\tau}:=\left\{a-\tau: a \in A_{\tau}^{\varepsilon_{1}}\right\}$. Furthermore, we can describe it in detail as follows:

(a) if for any $p>0$, there exists a number $P>p$ and $\tau \in E\left\{\mathbb{T}, \varepsilon_{1}\right\} \cap(P,+\infty)$, then we say $\mathbb{T}$ is a positivedirection ACCTS;

(b) if for any $\mathrm{q}<0$, there exists a number $\mathrm{Q}<\mathrm{q}$ and $\tau \in \mathrm{E}\left\{\mathbb{T}, \varepsilon_{1}\right\} \cap(-\infty, \mathrm{Q})$, then we say $\mathbb{T}$ is a negative-direction ACCTS;

(c) if for any $p>0, q<0$, there exist numbers $Q<q, P>p$ and $\pm \tau \in E\left\{\mathbb{T}, \varepsilon_{1}\right\} \cap((-\infty, Q) \cup(P,+\infty))$, then we say $\mathbb{T}$ is a bi-direction ACCTS;

(d) we say $\mathbb{T}$ is an oriented-direction ACCTS if $\mathbb{T}$ is a positive-direction ACCTS or a negative-direction ACCTS.

Remark 2.7. If $\pm \tau \in E\left\{\mathbb{T}, \varepsilon_{1}\right\}$ in Definition 2.6 from [33], then there exists $A_{ \pm \tau}^{\varepsilon_{1}}=\mathbb{T} \backslash \mathbb{T}^{ \pm \tau}$ such that $\mathrm{d}\left(\mathbb{T} \backslash A_{ \pm \tau}^{\varepsilon_{1}}, \mathbb{T}^{ \pm \tau}\right) \leqslant \mathrm{d}\left(\mathbb{T}, \mathbb{T}^{ \pm \tau}\right)<\varepsilon_{1}$, which implies that almost periodic time scales introduced in [30, 31] are bi-direction ACCTS with inf $\mathbb{T}=-\infty$, sup $\mathbb{T}=+\infty$. For more important comments and remarks of almost periodic time scales, one may consult $[3,33]$.

Remark 2.8. According to Definition 2.6, one can obtain that for any $\tau \in \Pi_{\varepsilon}$, it follows from $\mathrm{d}\left(\overline{\mathbb{T} \backslash A_{\tau}^{\varepsilon}}, \mathbb{T}^{\tau}\right)<$ $\varepsilon$ that $\mathrm{d}\left(\overline{\mathbb{T}^{-\tau} \backslash A_{-\tau}^{\varepsilon}}, \mathbb{T}\right)<\varepsilon$.

Let $\mu^{\tau}: \mathbb{T}^{\tau} \rightarrow \mathbb{R}^{+}$be the graininess function of $\mathbb{T}^{\tau}$ and $A_{\tau}$ be a set, satisfying

$$
\mu^{\tau}(t+\tau)= \begin{cases}\mu(t), & t+\tau \notin \overline{\mathbb{T} \backslash A_{\tau}}, \\ \mu(t+\tau), & t+\tau \in \overline{\mathbb{T} \backslash A_{\tau}} .\end{cases}
$$

Then, from (2.1), we can simplify Definition 2.6 as follows.

Definition 2.9. Let $\mu: \mathbb{T} \rightarrow \mathbb{R}, \mu(t)=\sigma(t)-t$. We say $\mathbb{T}$ is an almost-complete closedness time scale if for any $\varepsilon>0$, there exists the set $A_{-\tau}$ such that the set

$$
\Pi^{* *}=\left\{\tau \in \Pi:|\mu(\mathrm{t}+\tau)-\mu(\mathrm{t})|<\varepsilon, \forall \mathrm{t} \in \widetilde{\mathscr{R}}_{\mathbb{T}}(\tau)\right\}
$$

is relatively dense in $\Pi$, where $\widetilde{\mathscr{R}}_{\mathbb{T}}(\tau):=\mathbb{T} \cap\left(\cup_{\tau \in \Pi} \overline{\mathbb{T}^{-\tau} \backslash A_{-\tau}}\right), A_{-\tau}:=\left\{a-\tau: a \in A_{\tau}\right\}$. 
Remark 2.10. Since $\Pi^{* *}$ is relatively dense in $\Pi$ in Definition 2.9 , one can observe that the graininess function $\mu$ is bounded. From [33], we can see that ACCTS is the most general type of independent variables with almost periodicity.

We can also introduce an equivalent definition that depends on sequential convergence.

Definition 2.11. If a time scale $\mathbb{T}$ fulfills the following conditions:

(1) for any given sequence $\alpha^{\prime} \subset \Pi \cap(0,+\infty)$, there exist a subsequence $\alpha \subset \alpha^{\prime}$ and a sequence $\left\{A_{-\alpha_{n}}\right\}$ such that $\left\{\mathbb{T}^{-\alpha^{n}} \backslash A_{-\alpha_{n}}\right\}$ converges to a time scale $\mathbb{T}_{0}$, we say $\mathbb{T}$ is a positive-direction ACCTS;

(2) for any given sequence $\alpha^{\prime} \subset \Pi \cap(-\infty, 0)$, there exist a subsequence $\alpha \subset \alpha^{\prime}$ and a sequence $\left\{A_{-\alpha_{n}}\right\}$ such that $\left\{\mathbb{T}^{-\alpha^{n}} \backslash A_{-\alpha_{n}}\right\}$ converges to a time scale $\mathbb{T}_{0}$, we say $\mathbb{T}$ is a negative-direction ACCTS;

(3) for any given sequence $\alpha^{\prime} \subset \Pi$, there exist a subsequence $\alpha \subset \alpha^{\prime}$ and a sequence $\left\{A_{-\alpha_{n}}\right\}$ such that $\left\{\mathbb{T}^{-\alpha^{n}} \backslash A_{-\alpha_{n}}\right\}$ converges to a time scale $\mathbb{T}_{0}$, we say $\mathbb{T}$ is a bi-direction ACCTS;

(4) if $\mathbb{T}$ is a positive-direction ACCTS or a negative-direction ACCTS, we say $\mathbb{T}$ is an oriented-direction ACCTS.

Remark 2.12. Since the concept of almost periodic time scales from [33] is a particular case of Definition 2.6, if $\mathbb{T}$ is a bi-direction ACCTS (i.e., an almost periodic time scale), then $\mathscr{R}_{\mathbb{T}}(\tau, \varepsilon)=\mathbb{T} \cap\left(\cup \mathbb{T}^{\Pi_{\varepsilon}}\right)$, where $\mathbb{T} \cap\left(\cup \mathbb{T}^{\Pi_{\varepsilon}}\right)$ is the time variable set from Remark 5.4 in [30].

According to the concept of $\Delta$-sub-derivative (i.e., Definition 2.5 from [32]), we can introduce a concept of $\varepsilon$-equivalent impulsive evolution equations on ACCTS.

For any $s \in \Pi$ and a set $A_{-s}:=\left\{a-s: a \in A_{s}\right\}$ with $\mu_{\Delta}\left(A_{s}\right)>0$, let

$$
\mathfrak{B}^{s}=\left\{\left\{t_{k_{i}, s}\right\} \subset\left\{t_{k}\right\}: t_{k_{i}, s} \in \mathbb{T} \cap\left(\overline{\mathbb{T}^{-s} \backslash A_{-s}}\right), t_{k_{i}, s}<t_{k_{i+1}, s}, i \in \mathbb{Z}, \lim _{i \rightarrow \infty} t_{k_{i}, s}=\infty\right\},
$$

which denotes all unbounded increasing sequences of real numbers and

$$
\cup \mathfrak{B}^{s}=\left\{t_{k_{i}, s} \in\left\{t_{k}\right\}: t_{k_{i}, s} \in \mathbb{T} \cap\left(\overline{\mathbb{T}^{-s} \backslash A_{-s}}\right), t_{k_{i}, s}<t_{k_{i+1}, s}, i \in \mathbb{Z}, \lim _{i \rightarrow \infty} t_{k_{i}, s}=\infty\right\} .
$$

Definition 2.13. Let $\mathbb{T}$ be an almost-complete closedness time scale. Consider the following impulsive evolution equation with sub-derivative $x^{\Delta_{-s}}(t)$ on $\mathbb{T} \cap\left(\overline{\mathbb{T}^{-s} \backslash A_{-s}}\right)$ :

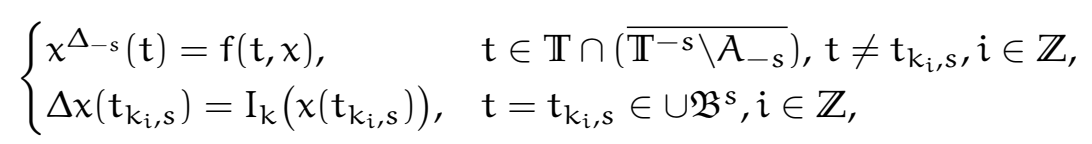

where $s \in \Pi$ and $f \in \mathrm{PC}_{\mathrm{rd}}(\mathbb{T} \times \mathbb{X}, \mathbb{X})$. We say the following impulsive evolution equation

$$
\begin{cases}x^{\Delta}(t)=f(t, x), & t \in \mathscr{R}_{\mathbb{T}}(s, \varepsilon), t \neq t_{k_{i}, s}, i \in \mathbb{Z}, \\ \Delta x\left(t_{k_{i}, s}\right)=I_{k}\left(x\left(t_{k_{i}, s}\right)\right), & t=t_{k_{i}, s} \in \cup \mathfrak{B}^{s}, i \in \mathbb{Z},\end{cases}
$$

is an $\varepsilon$-equivalent impulsive evolution equation for (2.2) if $s \in \Pi_{\varepsilon} \subset \Pi$.

\section{Weighted piecewise pseudo double-almost periodic mild solutions}

Throughout this paper, we shall assume that $\mathbb{T}$ is an almost-complete closedness time scale and denote by $\mathbb{X}$ a Banach space; let $\mathfrak{B}$ be the set consisting of all sequences $\left\{t_{k}\right\}_{k \in \mathbb{Z}}$ such that $\theta=\inf _{k \in \mathbb{Z}}\left(t_{k+1}-\right.$ $\left.t_{k}\right)>0$. For $\left\{t_{k}\right\}_{k \in \mathbb{Z}} \in \mathfrak{B}$, let $\mathrm{BPC}_{\mathrm{rd}}(\mathbb{T}, \mathbb{X})$ be the space formed by all bounded $r d$-piecewise continuous functions $\phi: \mathbb{T} \rightarrow \mathbb{X}$ such that $\phi(\cdot)$ is continuous at $t$ for any $t \notin\left\{t_{k}\right\}_{k \in \mathbb{Z}}$ and $\phi\left(t_{k}\right)=\phi\left(t_{k}^{-}\right)$for all $k \in \mathbb{Z}$; let $\Omega$ be a set of $\mathbb{X}$ and BPC $_{\mathrm{rd}}(\mathbb{T} \times \Omega, \mathbb{X})$ be the space formed by all bounded piecewise continuous functions $\phi: \mathbb{T} \times \Omega \rightarrow \mathbb{X}$ such that for any $x \in \Omega, \phi(\cdot, x) \in \mathrm{BPC}_{\mathrm{rd}}(\mathbb{T}, \mathbb{X})$ and for any $\mathrm{t} \in \mathbb{T}, \phi(t, \cdot)$ is continuous at $x \in \Omega$.

In the following, we give the definition of rd-piecewise continuous functions in Banach space on time scales. 
Definition 3.1. We say $\varphi: \mathbb{T} \rightarrow \mathbb{X}$ is rd-piecewise continuous with respect to a sequence $\left\{t_{k}\right\} \subset \mathbb{T}$ which satisfies $t_{k}<t_{k+1}, k \in \mathbb{Z}$, if $\varphi(t)$ is continuous on $\left[t_{k}, t_{k+1}\right)_{\mathbb{T}}$ and rd-continuous on $\mathbb{T} \backslash\left\{t_{k}\right\}$. Further, $\left[t_{k}, t_{k+1}\right)_{\mathbb{T}}$ are called intervals of continuity of the function $\varphi(t)$.

Similarly, one can define a class of ld-piecewise continuous functions. For convenience, we denote the space of all $\mathrm{rd}$-piecewise continuous functions $\mathrm{PC}_{\mathrm{rd}}(\mathbb{T}, \mathbb{X})$ and $\mathrm{PC}_{\mathrm{rd}}^{\varepsilon}(\mathbb{T}, \mathbb{X}):=\left\{\left.f\right|_{\mathscr{R}_{\mathbb{T}}(\tau, \varepsilon)}: f \in \mathrm{PC}_{\mathrm{rd}}(\mathbb{T}, \mathbb{X})\right\}$.

Now, we introduce some definitions which will be used to introduce the concept of weighted piecewise pseudo double-almost periodic functions on ACCTS. Let $\mathfrak{B}^{\varepsilon}=\left\{\left\{t_{k_{i}, \varepsilon}\right\} \subset\left\{t_{k}\right\}: t_{k_{i}} \in \mathscr{R}_{\mathbb{T}}(\tau, \varepsilon), t_{k_{i}, \varepsilon}<\right.$ $\left.t_{k_{i+1}, \varepsilon}, i \in \mathbb{Z}, \lim _{i \rightarrow \infty} t_{k_{i}, \varepsilon}=\infty\right\}$.

Definition 3.2. Let $\left\{t_{k_{i}, \varepsilon}\right\} \in \mathfrak{B}^{\varepsilon}, i \in \mathbb{Z}$. We say $\left\{t_{k_{i}, \varepsilon}^{j}\right\}$ is an $\varepsilon$-derived sequence of $\left\{t_{k_{i}, \varepsilon}\right\}$ where $t_{k_{i}, \varepsilon}^{j}=$ $t_{k_{i+j}, \varepsilon}-t_{k_{i}, \varepsilon}, i, j \in \mathbb{Z}$.

Definition 3.3. For any $\varepsilon_{2}>\varepsilon_{1}>0$, let $\Gamma \subset \Pi_{\varepsilon_{1}}$ be a set of real numbers and $\left\{\mathfrak{t}_{k_{i}, \varepsilon_{1}}\right\} \in \mathfrak{B}^{\varepsilon_{1}}$. We say $\left\{t_{k_{i}, \varepsilon_{1}}^{j}\right\}, i, j \in \mathbb{Z}$ is equipotentially double-almost periodic on an almost-complete closedness time scale $\mathbb{T}$ if for $r \in \Gamma$, there exists at least one integer q such that

$$
\left|t_{k_{i}, \varepsilon_{1}}^{q}-r\right|<\varepsilon_{2} \text { for all } i \in \mathbb{Z} \text {. }
$$

In the following, we introduce the concept of piecewise continuous double-almost periodic functions on ACCTS.

Definition 3.4. Let $\mathbb{T}$ be an almost-complete closedness time scale and assume that $\left\{\mathfrak{t}_{k_{i}, \varepsilon_{1}}\right\} \in \mathfrak{B}^{\varepsilon_{1}}$ satisfying the $\varepsilon_{1}$-derived sequence $\left\{t_{k_{i}, \varepsilon_{1}}^{j}\right\}, i, j \in \mathbb{Z}$, is equipotentially almost periodic. We call a function $\varphi \in$ $\mathrm{PC}_{\mathrm{rd}}^{\varepsilon_{1}}\left(\mathbb{T}, \mathbb{R}^{\mathrm{n}}\right)$ double-almost periodic if:

(i) for any $\varepsilon>0$, there is a positive number $\delta=\delta(\varepsilon)$ such that if the points $t^{\prime}$ and $t^{\prime \prime}$ belong to the same interval of continuity and $t^{\prime}, t^{\prime \prime} \in \mathscr{R}_{\mathbb{T}}\left(\tau, \varepsilon_{1}\right) \backslash \mathfrak{B}^{\varepsilon_{1}},\left|t^{\prime}-t^{\prime \prime}\right|<\delta$, then $\left\|\varphi\left(t^{\prime}\right)-\varphi\left(t^{\prime \prime}\right)\right\|<\varepsilon$;

(ii) for any $\varepsilon_{2}>\varepsilon_{1}>0$, there is a relative dense set $\Gamma$ of $\varepsilon_{2}$-almost periods such that if $\tau \in \Gamma \subset \Pi_{\varepsilon_{1}}$, then $\|\varphi(t+\tau)-\varphi(t)\|<\varepsilon_{2}$ for all $t \in \mathscr{R}_{\mathbb{T}}\left(\tau, \varepsilon_{1}\right)$ which satisfy the condition $\left|t-t_{k_{i}, \varepsilon_{1}}\right|>\varepsilon_{2}, i \in \mathbb{Z}$.

Remark 3.5. If we let $\mathbb{T}$ be a bi-direction ACCTS, then Definition 3.4 will become the concept of almost periodic functions from [33]. Further, if let $\mathbb{T}$ be a bi-direction periodic time scale, i.e., $\varepsilon_{1} \rightarrow 0$, then Definition 3.4 will become the concept of piecewise almost periodic functions on periodic time scales from [33]. For more properties and applications related to these functions and dynamic systems, one should consult [30, 31].

We denote by $\operatorname{DAP}(\mathbb{T}, \mathbb{X})$ the space of all rd-piecewise double-almost periodic functions. Obviously, for any fixed $\varepsilon>0$, the space $\operatorname{DAP}^{\varepsilon}(\mathbb{T}, \mathbb{X}):=\left\{\left.\mathbf{f}\right|_{\mathscr{R}}(\tau, \varepsilon): f \in \operatorname{DAP}(\mathbb{T}, \mathbb{X})\right\}$ endowed with norm $\|\phi\|_{\varepsilon}=$ $\sup _{t \in \mathscr{R}_{\mathbb{T}}(\tau, \varepsilon)}\|\phi(t)\|$ for any $\phi \in \operatorname{DAP}^{\varepsilon}(\mathbb{T}, \mathbb{X})$ is a Banach space. We also denote by UPC(T, $\left.\mathbb{X}\right)$ the space of all functions $\phi \in \mathrm{PC}_{\mathrm{rd}}(\mathbb{T}, \mathbb{X})$ such that $\phi$ satisfies the condition (i) in Definition 3.4 and $\mathrm{UPC}^{\varepsilon}(\mathbb{T}, \mathbb{X}):=$ $\left\{\left.f\right|_{\mathscr{R}_{\mathbb{T}}(\tau, \varepsilon)}: f \in \operatorname{UPC}(\mathbb{T}, \mathbb{X})\right\}$. Now $\mathrm{BPC}_{\mathrm{rd}}(\mathbb{T}, \mathbb{X})$ denotes the space of all bounded rd-piecewise functions and $\mathrm{BPC}_{\mathrm{rd}}^{\varepsilon}(\mathbb{T}, \mathbb{X}):=\left\{\left.f\right|_{\mathscr{R}_{\mathbb{T}}(\tau, \varepsilon)}: \mathrm{f} \in \mathrm{BPC}_{\mathrm{rd}}(\mathbb{T}, \mathbb{X})\right\}$.

Similarly, we can also introduce the concept of uniformly piecewise double-almost periodic functions on ACCTS as follows.

Definition 3.6. Let $\mathbb{T}$ be an almost-complete closedness time scale and assume that $\left\{\boldsymbol{t}_{\mathrm{k}_{i}, \varepsilon_{1}}\right\} \in \mathfrak{B}^{\varepsilon_{1}}$ satisfying the $\varepsilon_{1}$-derived sequence $\left\{t_{k_{i}, \varepsilon_{1}}^{j}\right\}, i, j \in \mathbb{Z}$, is equipotentially double-almost periodic. We call a function $f \in \mathrm{PC}_{\mathrm{rd}}^{\varepsilon_{1}}(\mathbb{T} \times \Omega, \mathbb{X}) \mathrm{rd}$-piecewise double-almost periodic in $t$ uniformly in $x \in \Omega$ if:

(i) for each compact set $\mathrm{K} \subseteq \Omega,\{\mathrm{f}(\cdot, \mathrm{x}): \mathrm{x} \in \mathrm{K}\}$ is uniformly bounded;

(ii) for any $\varepsilon>0$, there is a positive number $\delta=\delta(\varepsilon)$ such that if the points $t^{\prime}$ and $t^{\prime \prime}$ belong to the same interval of continuity and $t^{\prime}, t^{\prime \prime} \in \mathscr{R}_{\mathbb{T}}\left(\tau, \varepsilon_{1}\right) \backslash \mathfrak{B}^{\varepsilon_{1}},\left|t^{\prime}-t^{\prime \prime}\right|<\delta$, then $\left\|f\left(t^{\prime}, x\right)-f\left(t^{\prime \prime}, x\right)\right\|<\varepsilon$ for all $x \in \mathrm{K}$; 
(iii) for any $\varepsilon_{2}>\varepsilon_{1}>0$, there is relative dense set $\Gamma$ of $\varepsilon_{2}$-almost periods such that if $\tau \in \Gamma \subset \Pi_{\varepsilon_{1}}$, then $\|f(t+\tau, x)-f(t, x)\|<\varepsilon_{2}$ for all $t \in \mathscr{R}_{\mathbb{T}}\left(\tau, \varepsilon_{1}\right), x \in K$, which satisfy the condition $\left|t-t_{k_{i}, \varepsilon_{1}}\right|>\varepsilon_{2}, i \in$ $\mathbb{Z}$.

Now, let $\mathrm{U}$ be the set of all functions $\rho: \mathbb{T} \rightarrow(0, \infty)$ which are positive and locally $\Delta$-integrable over $\mathbb{T}$ and let $U^{\varepsilon}:=\left\{\left.\rho\right|_{\mathscr{R}_{\mathbb{T}}(\tau, \varepsilon)}: \rho \in U\right\}$. For a given $r_{1}, r_{2} \in \mathscr{R}_{\mathbb{T}}(\tau, \varepsilon), r_{2}>r_{1}$, we set

$$
\mathrm{m}\left(\mathrm{r}_{1}, \mathrm{r}_{2}, \rho\right):=\int_{\mathrm{r}_{1}}^{\mathrm{r}_{2}} \tilde{\rho}(\mathrm{s}) \Delta \mathrm{s}
$$

for each $\tilde{\rho} \in \mathrm{U}^{\varepsilon}$. Let $\mathrm{D}_{\mathrm{r}}:=\mathrm{r}_{2}-\mathrm{r}_{1}$ and $\tilde{\mathrm{U}}_{\infty}^{\varepsilon}:=\left\{\tilde{\rho} \in \mathrm{U}^{\varepsilon}: \lim _{\mathrm{D}_{\mathrm{r}} \rightarrow \infty} \mathrm{m}\left(\mathrm{r}_{1}, \mathrm{r}_{2}, \tilde{\rho}\right)=\infty\right\}$,

$$
\begin{aligned}
& \mathrm{U}_{\infty}^{\varepsilon}=\left\{\tilde{\rho} \in \tilde{\mathrm{U}}_{\infty}^{\varepsilon}: \tilde{\rho}(\mathrm{s}) \not \equiv 0 \text { for all } s \in(\mathrm{t}-\delta, \mathrm{t}+\delta)_{\mathscr{R}_{\mathbb{T}}(\tau, \varepsilon)} \text {, where } t \in \mathscr{R}_{\mathbb{T}}(\tau, \varepsilon), \delta>0\right\}, \\
& \mathrm{U}_{\mathrm{B}}^{\varepsilon}:=\left\{\tilde{\rho} \in \mathrm{U}_{\infty}^{\varepsilon}: \tilde{\rho} \text { is bounded and } \inf _{s \in \mathscr{R}_{\mathbb{T}}(\tau, \varepsilon)} \tilde{\rho}(\mathrm{s})>0\right\} .
\end{aligned}
$$

It is clear that for any $\varepsilon>0, \mathrm{U}_{\mathrm{B}}^{\varepsilon} \subset \mathrm{U}_{\infty}^{\varepsilon} \subset \mathrm{U}^{\varepsilon}$. Now, for $\tilde{\rho} \in \mathrm{U}_{\infty}^{\varepsilon}$, we define

$$
\operatorname{WPDAP}_{0}^{\varepsilon}(\mathbb{T}, \tilde{\rho}):=\left\{\phi \in \mathrm{BPC}_{\mathrm{rd}}^{\varepsilon}(\mathbb{T}, \mathbb{X}): \lim _{\mathrm{D}_{\mathrm{r}} \rightarrow \infty} \frac{1}{\mathrm{~m}\left(\mathrm{r}_{1}, \mathrm{r}_{2}, \tilde{\rho}\right)} \int_{\mathrm{r}_{1}}^{\mathrm{r}_{2}}\|\phi(s)\| \tilde{\rho}(\mathrm{s}) \Delta s=0\right\} .
$$

Similarly, we define

$$
\operatorname{WPDAP}_{0}^{\varepsilon}(\mathbb{T} \times \mathbb{X}, \tilde{\rho}):=\left\{\Phi \in \mathrm{BPC}_{\mathrm{rd}_{\mathrm{d}}}^{\varepsilon}(\mathbb{T} \times \Omega, \mathbb{X}): \lim _{\mathrm{D}_{\mathrm{r}} \rightarrow \infty} \frac{1}{\mathrm{~m}\left(\mathrm{r}_{1}, \mathrm{r}_{2}, \tilde{\rho}\right)} \int_{\mathrm{r}_{1}}^{r_{2}}\|\Phi(s, x)\| \tilde{\rho}(\mathrm{s}) \Delta s=0\right.
$$

uniformly with respect to $x \in K$, where $K$ is an arbitrary compact subset of $\Omega\}$.

We are now ready to introduce the sets $\operatorname{WPDAP}^{\varepsilon}(\mathbb{T}, \tilde{\rho})$ and $\operatorname{WPDAP}^{\varepsilon}(\mathbb{T} \times \mathbb{X}, \tilde{\rho})$ of weighted pseudo double-almost periodic functions on ACCTS:

$$
\begin{aligned}
\operatorname{WPDAP}^{\varepsilon}(\mathbb{T}, \tilde{\rho}) & =\left\{\mathrm{f}=\mathrm{g}+\phi \in \operatorname{PC}_{\mathrm{rd}}^{\varepsilon}(\mathbb{T}, \mathbb{X}): \mathrm{g} \in \operatorname{DAP}^{\varepsilon}(\mathbb{T}, \mathbb{X}) \text { and } \phi \in \operatorname{WPDAP}_{0}^{\varepsilon}(\mathbb{T}, \tilde{\rho})\right\}, \\
\operatorname{WPDAP}^{\varepsilon}(\mathbb{T} \times \mathbb{X}, \tilde{\rho}) & =\left\{\mathrm{f}=\mathrm{g}+\phi \in \operatorname{PC}_{\mathrm{rd}}^{\varepsilon}(\mathbb{T} \times \mathbb{X}, \mathbb{X}): \mathrm{g} \in \operatorname{DAP}^{\varepsilon}(\mathbb{T} \times \mathbb{X}, \mathbb{X}) \text { and } \phi \in \operatorname{WPDAP}_{0}^{\varepsilon}(\mathbb{T} \times \mathbb{X}, \tilde{\rho})\right\} .
\end{aligned}
$$

In what follows, through the established results from [34], we can establish some theoretical results for the existence and stability of weighted piecewise pseudo double-almost periodic mild solutions for Eq. (1.1). For this, we need the following lemma.

Lemma 3.7. Let $\mathbb{T}$ be an almost-complete closedness time scale and $\ominus \omega \in \mathcal{R}^{+}$. For all $\mathrm{t} \in \mathscr{R}_{\mathbb{T}}(\tau, \varepsilon)$ and $\alpha \in \Pi_{\varepsilon}$, there exist constants $\beta_{1}, \beta_{2}>0$ such that

$$
\beta_{1} \mu(t) \leqslant \mu(t+\alpha) \leqslant \beta_{2} \mu(t) .
$$

Then, there exist positive constants $\mathrm{K}^{*}$ and $\omega^{*}$ such that

$$
e_{\ominus \omega}(t+\alpha, s+\alpha) \leqslant K^{*} e_{\ominus \omega^{*}}(t, s), t \geqslant s, t, s \in \mathscr{R}_{\mathbb{T}}(\tau, \varepsilon) .
$$

Proof. Obviously, if $\mu=0, \mathbb{T}=\mathbb{R}$, the result holds. Assume that $\mu \not \equiv 0$. Since $\ominus \omega \in \mathcal{R}^{+}$, we have

$$
e_{\ominus \omega}(t+\alpha, s+\alpha)=\exp \left\{\int_{s+\alpha}^{t+\alpha} \frac{1}{\mu(\tau)} \ln \frac{1}{1+\mu(\tau) \omega} \Delta \tau\right\}=\exp \left\{\int_{s}^{t} \frac{1}{\mu(\tau+\alpha)} \ln \frac{1}{1+\mu(\tau+\alpha) \omega} \Delta \tau\right\} .
$$

Since $\mathbb{T}$ is an almost-complete closedness time scale, $\mu$ is bounded. Hence, from the inequality (3.1), for $\mathrm{t}, \mathrm{s} \in \mathscr{R}_{\mathbb{T}}(\alpha, \varepsilon), \mathrm{t} \geqslant \mathrm{s}$, we obtain

$$
e_{\ominus \omega}(t+\alpha, s+\alpha) \leqslant \exp \left\{\int_{s}^{t} \frac{1}{\beta_{1} \mu(\tau)} \ln \frac{1}{1+\beta_{1} \mu(\tau) \omega} \Delta \tau\right\}=\left\{\exp \left\{\int_{s}^{t} \frac{\ln \left(1+\mu(\tau)\left(\ominus \beta_{1} \omega\right)\right)}{\mu(\tau)}\right\}\right\}^{\frac{1}{\beta_{1}}}
$$


Thus, there exists a positive constant $K^{*}>0$ such that

$$
e_{\ominus \omega}(t+\alpha, s+\alpha)=\left[e_{\ominus \beta_{1} \omega}(t, s)\right]^{\frac{1}{\beta_{1}}} \leqslant K^{*} e_{\ominus \omega^{*}}(t, s),
$$

where $\omega^{*}=\beta_{1} \omega$. This completes the proof.

Let $\mathbb{T}$ be an almost-complete closedness time scale, and consider the linear evolution system

$$
x^{\Delta}=A(t) x, t \in \mathscr{R}_{\mathbb{T}}(\tau, \varepsilon),
$$

where $A: \mathscr{R}_{\mathbb{T}}(\tau, \varepsilon) \rightarrow B(\mathbb{X})$ is a linear operator in the Banach space $\mathbb{X}$. We denote by $\mathrm{B}(\mathbb{X}, \mathbb{Y})$ the Banach space of all bounded linear operators from $\mathbb{X}$ to $\mathbb{Y}$. This is denoted as $B(\mathbb{X})$ when $\mathbb{X}=\mathbb{Y}$.

Inspired by [22], we present Definitions 3.8-3.9.

Definition 3.8. Let $\mathbb{T}$ be an almost-complete closedness time scale. Now $\mathrm{T}(\mathrm{t}, \mathrm{s}): \mathscr{R}_{\mathbb{T}}(\tau, \varepsilon) \times \mathscr{R}_{\mathbb{T}}(\tau, \varepsilon) \rightarrow$ $\mathrm{B}(\mathbb{X})$ is called the linear evolution operator associated to (3.2) if $\mathrm{T}(\mathrm{t}, \mathrm{s})$ satisfies the following conditions:

(1) $\mathrm{T}(\mathrm{s}, \mathrm{s})=\mathrm{Id}$, where Id denotes the identity operator in $\mathbb{X}$;

(2) $\mathrm{T}(\mathrm{t}, \mathrm{s}) \mathrm{T}(\mathrm{s}, \mathrm{r})=\mathrm{T}(\mathrm{t}, \mathrm{r})$;

(3) the mapping $(t, s) \rightarrow T(t, s) x$ is continuous for any fixed $x \in \mathbb{X}$.

Definition 3.9. An evolution operator $T(t, s)$ is called exponentially stable on an almost-complete closedness $\mathbb{T}$ if there exists $K_{0} \geqslant 1$ and $\omega>0$ such that

$$
\|T(t, s)\|_{B(\mathbb{X})} \leqslant K_{0} e_{\ominus \omega}(t, s), t \geqslant s, t, s \in \mathscr{R}_{\mathbb{T}}(\tau, \varepsilon) .
$$

Definition 3.10. A function $x: \mathbb{T} \rightarrow \mathbb{X}$ is called an $\varepsilon$-mild solution of Eq. (1.1) if for any $t \in \mathscr{R}_{\mathbb{T}}(\tau, \varepsilon), t>$ $c, c \neq t_{k_{i}, \varepsilon}, i \in \mathbb{Z}$,

$$
x(t)=T(t, c) x(c)+\int_{c}^{t} T(t, \sigma(s)) f(s, x(s)) \Delta s+\sum_{c<t_{k_{i}, \varepsilon}<t} T\left(t, t_{k_{i}, \varepsilon}\right) I_{k}\left(x\left(t_{k_{i}, \varepsilon}\right)\right) .
$$
tions.

In the following, we shall consider the abstract differential system (1.1) with the following assump-

$\left(H_{1}\right)$ The family $\left\{A(t): t \in \mathscr{R}_{\mathbb{T}}\left(\tau, \varepsilon_{1}\right)\right\}$ of operators in $\mathbb{X}$ generates an exponentially stable evolution system $\{T(t, s): t \geqslant s\}$, i.e., there exist $K_{0}>1$ and $\omega>0$ such that

$$
\|\mathrm{T}(\mathrm{t}, \mathrm{s})\|_{\mathrm{B}(\mathbb{X})} \leqslant \mathrm{K}_{0} e_{\ominus \omega}(\mathrm{t}, \mathrm{s}), \mathrm{t} \geqslant s, \mathrm{t}, \mathrm{s} \in \mathscr{R}_{\mathbb{T}}\left(\tau, \varepsilon_{1}\right)
$$

and for any $\varepsilon_{2}>\varepsilon_{1}>0$, there exists a relatively dense set $\bar{T} \subset \Pi_{\varepsilon_{1}}$ such that if $\tau \in \bar{T}$, then

$$
\|\mathrm{T}(\mathrm{t}+\tau, s+\tau)-\mathrm{T}(\mathrm{t}, \mathrm{s})\|_{\mathrm{B}(\mathbb{X})}<\varepsilon_{2} e_{\ominus \omega}(t, s), \mathrm{t} \geqslant s, \mathrm{t}, \mathrm{s} \in \mathscr{R}_{\mathrm{T}}\left(\tau, \varepsilon_{1}\right) .
$$

$\left(H_{2}\right) f=g+\phi \in \operatorname{WPDAP}^{\varepsilon}(\mathbb{T}, \tilde{\rho})$, where $\rho \in U_{\infty}^{\varepsilon}$ and $f(t, \cdot)$ is uniformly continuous in each bounded subset of $\Omega$ uniformly in $t \in \mathscr{R}_{\mathbb{T}}(\tau, \varepsilon) ;\left\{\mathrm{I}_{\mathrm{k}}\right\}$ is a weighted pseudo-almost periodic sequence, $\mathrm{I}_{\mathrm{k}}(\mathrm{x})$ is uniformly continuous in $x \in \Omega$ uniformly in $k \in \mathbb{Z}$, inf $_{i \in \mathbb{Z}} t_{k_{i}, \varepsilon}^{1}=\theta_{\varepsilon}>0$.

$\left(\mathrm{H}_{3}\right) \mathbb{X}$ is a finite dimensional Banach space.

$\left(\mathrm{H}_{4}\right)$ For any $\mathrm{L}>0$, let

$$
\mathrm{H}_{1 \mathrm{~L}}=\sup _{\mathrm{t} \in \mathscr{R}_{\mathbb{T}}\left(\tau, \varepsilon_{1}\right),\|x\|_{\varepsilon_{1}} \leqslant \mathrm{~L}}\|\mathrm{f}(\mathrm{t}, \mathrm{x})\|<+\infty \text { and } \mathrm{H}_{2 \mathrm{~L}}=\sup _{\|x\|_{\varepsilon_{1}} \leqslant \mathrm{~L}}\left\|\mathrm{I}_{\mathrm{k}}(x)\right\|<+\infty .
$$

Moreover, there exists a number $\mathrm{L}_{0}>0$ such that

$$
\frac{\mathrm{H}_{1 \mathrm{~L}_{0}} \mathrm{~K}_{0}\left(1+\left(\bar{\mu}+\varepsilon_{1}\right) \omega\right)}{\omega} \mathrm{H}_{1 \mathrm{~L}_{0}}+\frac{\mathrm{H}_{2 \mathrm{~L}_{0}} \mathrm{~K}_{0}}{1-e_{\ominus \omega}\left(\theta_{\varepsilon_{1}}, 0\right)} \leqslant \mathrm{L}_{0},
$$

where $\bar{\mu}=\sup _{t \in \mathbb{T}} \mu$ and $e_{\ominus \omega}\left(\theta_{\varepsilon_{1}}, 0\right):=\sup _{i \in \mathbb{Z}} e_{\ominus \omega}\left(t_{k_{i+1}, \varepsilon_{1}}, t_{k_{i}, \varepsilon_{1}}\right)$. 
Remark 3.11. It is obvious that if assumption $\left(H_{1}\right)$ holds, then for any sequence $\left\{s_{n}\right\}_{n=1}^{\infty} \subset \Pi_{\varepsilon}$, there exist a subsequence $\left\{s_{n_{\mathfrak{m}}}\right\}_{\mathfrak{m}=1}^{\infty} \subset\left\{s_{\mathfrak{n}}\right\}_{\mathfrak{n}=1}^{\infty}$ and a set sequence $\left\{A_{-s_{n_{m}}}\right\}$ such that the limit set $\mathbb{T}_{0}$ of $\left\{\mathbb{T}^{-s_{n_{m}} \backslash A} A_{-s_{n_{m}}}\right\}$ exists and $\lim _{m \rightarrow \infty} T\left(t+s_{n_{m}}, s+s_{n_{m}}\right)$ uniformly exists on $\mathbb{T}_{0}$, i.e., $T\left(t+s_{n_{m}}, s+s_{n_{m}}\right) \rightarrow T^{*}(t, s), m \rightarrow \infty$, for all $t, s \in \mathbb{T}_{0}$.

Lemma 3.12. In Remark 3.11, if $\left(\mathrm{H}_{1}\right)$ holds, then there exist positive constants $\mathrm{K}^{* *}$ and $\omega^{*}$ such that

$$
\left\|T^{*}(t, s)\right\|_{B(\mathbb{X})} \leqslant K^{* *} e_{\ominus \omega^{*}}(t, s), t \geqslant s, t, s \in \mathscr{R}_{\mathbb{T}}\left(\tau, \varepsilon_{1}\right),
$$

and for any $\varepsilon_{2}>\varepsilon_{1}>0, \tau \in \overline{\mathrm{T}} \subset \Pi_{\varepsilon_{1}}$,

$$
\left\|T^{*}(t+\tau, s+\tau)-T^{*}(t, s)\right\|_{B(\mathbb{X})} \leqslant \varepsilon_{2} K^{*} e_{\ominus \omega^{*}}(t, s), t \geqslant s, t, s \in \mathscr{R}_{\mathbb{T}}\left(\tau, \varepsilon_{1}\right) .
$$

Proof. Denote $\mathrm{T}\left(\mathrm{t}+\mathrm{s}_{\mathrm{n}_{\mathrm{m}}}, \mathrm{s}+\mathrm{s}_{\mathrm{n}_{\mathrm{m}}}\right)$ by $\mathrm{T}_{\mathrm{m}}(t, s)$. By Lemma 3.7, there exist positive constants $K^{*}$ and $\omega^{*}$ such that

$$
\left\|T_{m}(t, s)\right\|_{B(\mathbb{X})} \leqslant K_{0} e_{\ominus \omega}\left(t+s_{n_{m}}, s+s_{n_{m}}\right) \leqslant K_{0} K^{*} e_{\ominus \omega^{*}}(t, s), t \geqslant s,
$$

and for any $\varepsilon_{2}>\varepsilon_{1}>0, \tau \in \overline{\mathrm{T}} \subset \Pi_{\varepsilon_{1}}$,

$$
\left\|T_{m}(t, s)-T_{m}(t+\tau, s+\tau)\right\|_{B(X)} \leqslant \varepsilon_{2} e_{\ominus \omega}\left(t+s_{n_{m}}, s+s_{n_{m}}\right) \leqslant \varepsilon_{2} K^{*} e_{\ominus \omega^{*}}(t, s) .
$$

In (3.4), let $\mathrm{m} \rightarrow \infty$, so that

$$
\left\|T^{*}(t+\tau, s+\tau)-T^{*}(t, s)\right\|_{B(\mathbb{X})} \leqslant \varepsilon_{2} K^{*} e_{\ominus w^{*}}(t, s) .
$$

Also, by (3.4), we have

$$
\left\|T_{m}(t+\tau, s+\tau)-T^{*}(t, s)\right\|_{B(\mathbb{X})} \leqslant \varepsilon_{2} K^{*} e_{\ominus \omega^{*}}(t, s),
$$

thus, by (3.3) and (3.4), we have

$$
\begin{aligned}
\left\|T^{*}(t, s)\right\|_{B(\mathbb{X})} & =\left\|T_{m}(t+\tau, s+\tau)-T^{*}(t, s)-T_{m}(t+\tau, s+\tau)\right\|_{B(\mathbb{X})} \\
& \leqslant\left\|T_{m}(t+\tau, s+\tau)-T^{*}(t, s)\right\|_{B(X)}+\left\|T_{m}(t+\tau, s+\tau)\right\|_{B(\mathbb{X})} \\
& \leqslant \varepsilon_{2} K^{*} e_{\ominus \omega^{*}}(t, s)+K_{0} K^{*} e_{\ominus \omega^{*}}(t, s) \\
& =\left(\varepsilon_{2} K^{*}+K_{0} K^{*}\right) e_{\ominus \omega^{*}}(t, s):=K^{* *} e_{\ominus w^{*}}(t, s), t \geqslant s .
\end{aligned}
$$

This completes the proof.

To investigate the existence and uniqueness of a weighted pseudo double-almost periodic solution for Eq. (1.1), we need the following lemma:

Lemma 3.13. Let $v \in \operatorname{DAP}^{\varepsilon}(\mathbb{T}, \mathbb{X}), \mu \in \operatorname{DAP}^{\varepsilon}\left(\mathbb{T}, \mathbb{R}^{+}\right)$and $\left(\mathrm{H}_{1}\right)-\left(\mathrm{H}_{2}\right)$ be satisfied. If $u: \mathscr{R}_{\mathbb{T}}(\tau, \varepsilon) \rightarrow \mathbb{X}$ is defined by

$$
\mathrm{u}_{0}(\mathrm{t})=\int_{-\infty}^{\mathrm{t}} \mathrm{T}(\mathrm{t}, \sigma(\mathrm{s})) v(\mathrm{~s}) \Delta \mathrm{s}+\sum_{\mathrm{t}_{\mathrm{k}_{\mathrm{i}}, \varepsilon}<\mathrm{t}} \mathrm{T}\left(\mathrm{t}, \mathrm{t}_{\mathrm{k}_{\mathrm{i}}, \varepsilon}\right) \mathrm{I}_{\mathrm{k}}\left(v\left(\mathrm{t}_{\mathrm{k}_{\mathrm{i}}, \varepsilon}\right)\right), \mathrm{t} \geqslant \mathrm{s},
$$

then $\mathrm{u}_{0}(\cdot) \in \operatorname{DAP}^{\varepsilon}(\mathbb{T}, \mathbb{X})$.

Proof. Let $\left\{s_{\mathfrak{n}}\right\}_{\mathfrak{n}=1}^{\infty} \subset \Pi_{\mathcal{\varepsilon}}$. Since $v$ is double-almost periodic, there exist a subsequence $\left\{\tau_{\mathfrak{n}}\right\}_{\mathfrak{n}=1}^{\infty} \subset\left\{s_{\mathfrak{n}}\right\}_{\mathfrak{n}=1}^{\infty}$ and a set sequence $\left\{A_{-\tau_{n}}\right\}$ such that the limit set $\mathbb{T}_{0}$ of $\left\{\mathbb{T}^{-\tau_{n}} \backslash A_{-\tau_{n}}\right\}$ exists and $h(t):=\lim _{n \rightarrow \infty} v\left(t+\tau_{n}\right)$ uniformly exists for $t \in \mathbb{T}_{0}$. Now, we consider

$$
\begin{aligned}
u\left(t+\tau_{n}\right) & =\int_{-\infty}^{t+\tau_{n}} T\left(t+\tau_{n}, \sigma(s)\right) v(s) \Delta s \\
& =\int_{-\infty}^{t} T\left(t+\tau_{n}, \sigma\left(s+\tau_{n}\right)\right) v\left(s+\tau_{n}\right) \Delta s
\end{aligned}
$$




$$
=\int_{-\infty}^{t} \mathrm{~T}\left(\mathrm{t}+\tau_{\mathrm{n}}, \sigma\left(s+\tau_{\mathrm{n}}\right)\right) v_{\mathrm{n}}(\mathrm{s}) \Delta \mathrm{s}, \text { where } v_{\mathrm{n}}(\mathrm{s})=v\left(\mathrm{~s}+\tau_{\mathrm{n}}\right), \mathrm{n}=1,2, \ldots
$$

Note that $e_{\ominus \omega^{*}}(t, s)\left(1+\mu(s) \omega^{*}\right)=e_{\ominus \omega^{*}}(t, \sigma(s))$, so we have $e_{\ominus \omega^{*}}(t, s) \leqslant e_{\ominus \omega^{*}}(t, \sigma(s))$, and further, let $\mu_{\varepsilon}: \mathscr{R}_{\mathbb{T}}\left(\tau, \varepsilon_{1}\right) \rightarrow \mathbb{R}^{+}$, thus, we have $\left|\mu(\mathrm{t})-\mu_{\varepsilon}(\mathrm{t})\right|<\varepsilon$ for $\mathrm{t} \in \mathscr{R}_{\mathbb{T}}(\tau, \varepsilon)$. Thus, by $\left(\mathrm{H}_{1}\right)$ and Lemma 3.7, we obtain

$$
\begin{aligned}
\left\|u\left(t+\tau_{n}\right)\right\| & \leqslant \int_{-\infty}^{t}\left\|T\left(t+\tau_{n}, \sigma\left(s+\tau_{n}\right)\right) v_{n}(s)\right\| \Delta s \\
& \leqslant \int_{-\infty}^{t} K_{0} e_{\ominus \omega}\left(t+\tau_{n}, \sigma\left(s+\tau_{n}\right)\right)\left\|v_{n}(s)\right\| \Delta s \\
& \leqslant K_{0} \int_{-\infty}^{t}\left(1+\mu_{\varepsilon}(t)\right) e_{\ominus \omega}\left(t+\tau_{n}, s+\tau_{n}\right)\left\|v_{n}(s)\right\| \Delta s \\
& \leqslant(1+(\bar{\mu}+\varepsilon) \omega) K_{0} K^{*}\|v\|_{\varepsilon} \int_{-\infty}^{t} e_{\ominus \omega^{*}}(t, \sigma(s)) \Delta s \\
& =\frac{(1+(\bar{\mu}+\varepsilon) \omega) K_{0} K^{*}\|v\|_{\varepsilon}}{\ominus \omega^{*}}\left[e_{\ominus \omega^{*}}(t,-\infty)-e_{\ominus \omega^{*}}(t, t)\right] \\
& =\frac{K_{0} K^{*}\|v\|_{\varepsilon}(1+(\bar{\mu}+\varepsilon) \omega)\left(1+(\bar{\mu}+\varepsilon) \omega^{*}\right)}{\omega^{*}}
\end{aligned}
$$

where $\bar{\mu}=\sup _{t \in \mathbb{T}} \mu(t)$.

On the other hand, since $\mu \in \operatorname{DAP}^{\varepsilon}\left(\mathbb{T}, \mathbb{R}^{+}\right)$, we have $\mu\left(t+\tau_{n}\right) \rightarrow \mu^{*}(t), n \rightarrow \infty$, i.e., $\sigma\left(t+\tau_{n}\right)-\tau_{n} \rightarrow$ $\sigma^{*}(t), n \rightarrow \infty$, thus $\sigma\left(t+\tau_{n}\right) \rightarrow \sigma^{*}(t)+\tau_{n}, n \rightarrow \infty$. Therefore, by the condition (3) in Definition 3.8, we have

$$
\mathrm{T}\left(\mathrm{t}+\tau_{\mathrm{n}}, \sigma\left(\mathrm{s}+\tau_{\mathrm{n}}\right)\right) \rightarrow \mathrm{T}\left(\mathrm{t}+\tau_{\mathrm{n}}, \sigma^{*}(\mathrm{~s})+\tau_{\mathrm{n}}\right) \rightarrow \mathrm{T}^{*}\left(\mathrm{t}, \sigma^{*}(\mathrm{~s})\right), \mathrm{t}, \mathrm{s} \in \mathscr{R}_{\mathbb{T}}(\tau, \varepsilon), \mathrm{n} \rightarrow \infty .
$$

Further, it is easy to see that $v_{n}(s) \rightarrow h(s)$ as $n \rightarrow \infty$, for all $s \in \mathscr{R}_{\mathbb{T}}(\tau, \varepsilon)$. Thus, for any $t \geqslant s$, Lebesgue's dominated convergence theorem gives

$$
\lim _{n \rightarrow \infty} u\left(t+\tau_{n}\right)=\int_{-\infty}^{t} T^{*}\left(t, \sigma^{*}(s)\right) h(s) \Delta s .
$$

Next, we consider

$$
\begin{aligned}
u^{\prime}\left(t+\tau_{n}\right) & =\sum_{t_{k_{i}, \varepsilon}<t+\tau_{n}} T\left(t+\tau_{n}, t_{k_{i}, \varepsilon}\right) I_{k}\left(v\left(t_{k_{i}, \varepsilon}\right)\right) \\
& =\sum_{t_{k_{i}, \varepsilon}<t} T\left(t+\tau_{n}, t_{k_{i}, \varepsilon}+\tau_{n}\right) I_{k}\left(v\left(t_{k_{i}, \varepsilon}+\tau_{n}\right)\right)=\sum_{t_{k_{i}, \varepsilon}<t} T\left(t+\tau_{n}, t_{k_{i}, \varepsilon}+\tau_{n}\right) I_{k}\left(v_{i n}\right),
\end{aligned}
$$

where $v\left(t_{k_{i}, \varepsilon}+\tau_{n}\right):=v_{i n}$. By Lemma 3.7, we have

$$
\begin{aligned}
\left\|u^{\prime}\left(t+\tau_{n}\right)\right\| & =\left\|\sum_{t_{k_{i}, \varepsilon}<t+\tau_{n}} T\left(t+\tau_{n}, t_{k_{i}, \varepsilon}\right) I_{k}\left(v\left(t_{k_{i}, \varepsilon}\right)\right)\right\|=\left\|\sum_{t_{k_{i}, \varepsilon}<t} T\left(t+\tau_{n}, t_{k_{i}, \varepsilon}+\tau_{n}\right) I_{k}\left(v_{i n}\right)\right\| \\
& \leqslant I_{0} \sum_{t_{k_{i}, \varepsilon}<t} e_{\ominus \omega}\left(t+\tau_{n}, t_{k_{i}, \varepsilon}+\tau_{n}\right) \leqslant I_{0} K^{*} \sum_{t_{k_{i}, \varepsilon}<t} e_{\ominus \omega^{*}}\left(t, t_{k_{i}, \varepsilon}\right) \leqslant \frac{I K_{0} K^{*}}{1-e_{\ominus \omega^{*}}\left(\theta_{\varepsilon}, 0\right)} .
\end{aligned}
$$

Since $v \in \operatorname{DAP}^{\varepsilon}(\mathbb{T}, \mathbb{X}), v_{i n} \rightarrow h\left(t_{k_{i}, \varepsilon}\right), n \rightarrow \infty$, for all $i \in \mathbb{Z}$. Hence, for any $t>t_{k_{i}, \varepsilon}, i \in \mathbb{Z}$, by Lebesgue's dominated convergence theorem, we get

$$
\lim _{n \rightarrow \infty} u^{\prime}\left(t+\tau_{n}\right)=\sum_{t_{k_{i}, \varepsilon}<t} T^{*}\left(t, t_{k_{i}, \varepsilon}\right) I_{k}\left(h\left(t_{k_{i}, \varepsilon}\right)\right) .
$$


Thus, it follows that

$$
\lim _{n \rightarrow \infty} u_{0}\left(t+\tau_{n}\right)=\lim _{n \rightarrow \infty} u\left(t+\tau_{n}\right)+\lim _{n \rightarrow \infty} u^{\prime}\left(t+\tau_{n}\right)
$$

uniformly exists on $\mathbb{T}_{0}$. Therefore, $\mathrm{u}_{0}(\cdot) \in \operatorname{DAP}^{\varepsilon}(\mathbb{T}, \mathbb{X})$. This completes the proof.

Theorem 3.14. Let $\mathrm{f}(\cdot, \vartheta(\cdot)) \in \operatorname{WPDAP}^{\varepsilon}(\mathbb{T}, \tilde{\rho})$, where $\vartheta \in \operatorname{WPDAP}^{\varepsilon}(\mathbb{T}, \tilde{\rho})$ and $\{\mathrm{T}(\mathrm{t}, \mathrm{s}), \mathrm{t} \geqslant \mathrm{s}\}$ is exponentially stable, $\rho \in \mathrm{U}_{\infty}^{\varepsilon}$. Then, for all $\mathrm{t} \in \mathscr{R}_{\mathbb{T}}(\tau, \varepsilon)$,

$$
\mathrm{F}(\cdot):=\int_{-\infty}^{(\cdot)} \mathrm{T}(\cdot, \sigma(s)) f(s, \vartheta(s)) \Delta s+\sum_{t_{k_{i}, \varepsilon}<\cdot} \mathrm{T}\left(\cdot, t_{k_{i}, \varepsilon}\right) I_{k}\left(\vartheta\left(t_{k_{i}, \varepsilon}\right)\right) \in \operatorname{WPDAP}^{\varepsilon}(\mathbb{T}, \tilde{\rho}) .
$$

Proof. Fix $\vartheta \in \operatorname{WPDAP}^{\varepsilon}(\mathbb{T}, \mathbb{X})$, then we have $f(\cdot, \vartheta(\cdot))=\phi_{1}(\cdot)+\phi_{2}(\cdot)$, where $\phi_{1} \in \operatorname{DAP}^{\varepsilon}(\mathbb{T}, \mathbb{X}), \phi_{2} \in$ $\operatorname{WPDAP}_{0}^{\varepsilon}(\mathbb{T}, \mathbb{X})$. Thus,

$$
\int_{-\infty}^{\mathrm{t}} \mathrm{T}(\mathrm{t}, \sigma(\mathrm{s})) \mathrm{f}(\mathrm{s}, \vartheta(\mathrm{s})) \Delta s=\int_{-\infty}^{\mathrm{t}} \mathrm{T}(\mathrm{t}, \sigma(\mathrm{s})) \phi_{1}(\mathrm{~s}) \Delta \mathrm{s}+\int_{-\infty}^{\mathrm{t}} \mathrm{T}(\mathrm{t}, \sigma(\mathrm{s})) \phi_{2}(\mathrm{~s}) \Delta s:=\mathrm{I}_{1}(\mathrm{t})+\mathrm{I}_{2}(\mathrm{t})
$$

and

$$
\sum_{t_{k_{i}, \varepsilon}<t} T\left(t, t_{k_{i}, \varepsilon}\right) I_{k}\left(\vartheta\left(t_{k_{i}, \varepsilon}\right)\right)=\sum_{t_{k_{i}, \varepsilon}<t} T\left(t, t_{k_{i}, \varepsilon}\right) \beta_{i}+\sum_{t_{k_{i}, \varepsilon}<t} T\left(t, t_{k_{i}, \varepsilon}\right) \gamma_{i}:=\Upsilon_{1}(t)+\Upsilon_{2}(t)
$$

By Lemma 3.13, it is clear that $\mathrm{I}_{1}, \Upsilon_{1} \in \operatorname{DAP}^{\mathcal{E}}(\mathbb{T}, \mathbb{X})$. Moreover, it follows from Theorem 2.15 in [6] and $\left(\mathrm{H}_{1}\right)$ that

$$
\begin{aligned}
& \frac{1}{m\left(r_{1}, r_{2}, \tilde{\rho}\right)} \int_{r_{1}}^{r_{2}}\left\|I_{2}(t)\right\| \Delta t=\frac{1}{m\left(r_{1}, r_{2}, \tilde{\rho}\right)} \int_{r_{1}}^{r_{2}}\left\|\int_{-\infty}^{t} T(t, \sigma(s)) \phi_{2}(s) \Delta s\right\| \Delta t \\
& \leqslant \frac{1}{m\left(r_{1}, r_{2}, \tilde{\rho}\right)} \int_{r_{1}}^{r_{2}} \Delta t \int_{-\infty}^{t} K_{0} e_{\ominus \omega}(t, \sigma(s))\left\|\phi_{2}(s)\right\| \Delta s \\
& =\frac{1}{m\left(r_{1}, r_{2}, \tilde{\rho}\right)} \int_{r_{1}}^{r_{2}} \Delta t\left(\int_{-\infty}^{r_{1}} K_{0} e_{\ominus \omega}(t, \sigma(s))\left\|\phi_{2}(s)\right\| \Delta s\right. \\
& \left.+\int_{\mathrm{r}_{1}}^{\mathrm{t}} \mathrm{K}_{0} e_{\ominus \omega}(\mathrm{t}, \sigma(\mathrm{s}))\left\|\phi_{2}(\mathrm{~s})\right\| \Delta \mathrm{s}\right) \\
& =\frac{1}{\mathrm{~m}\left(\mathrm{r}_{1}, \mathrm{r}_{2}, \tilde{\rho}\right)} \int_{-\infty}^{\mathrm{r}_{1}}\left\|\phi_{2}(\mathrm{~s})\right\| \Delta s \int_{\mathrm{r}_{1}}^{\mathrm{r}_{2}} \mathrm{~K}_{0} e_{\ominus \omega}(\mathrm{t}, \sigma(\mathrm{s})) \Delta s \\
& +\frac{1}{\mathrm{~m}\left(\mathrm{r}_{1}, \mathrm{r}_{2}, \tilde{\rho}\right)} \int_{\mathrm{r}_{1}}^{\mathrm{r}_{2}}\left\|\phi_{2}(\mathrm{~s})\right\| \Delta s \int_{s}^{\mathrm{r}_{2}} \mathrm{~K}_{0} e_{\ominus \omega}(\mathrm{t}, \sigma(\mathrm{s})) \Delta \mathrm{t}:=\mathrm{I}_{1}^{0}+\mathrm{I}_{2}^{0} \text {. }
\end{aligned}
$$

Clearly,

$$
\begin{aligned}
& \mathrm{I}_{1}^{0}=\frac{1}{\mathrm{~m}\left(\mathrm{r}_{1}, \mathrm{r}_{2}, \tilde{\rho}\right)} \int_{-\infty}^{\mathrm{r}_{1}}\left\|\phi_{2}(\mathrm{~s})\right\| \Delta s \int_{\mathrm{r}_{1}}^{\mathrm{r}_{2}} \mathrm{~K}_{0} e_{\ominus \omega}(\mathrm{t}, \sigma(\mathrm{s})) \Delta \mathrm{t} \\
& =\frac{1}{m\left(r_{1}, r_{2}, \tilde{\rho}\right)} \int_{-\infty}^{r_{1}}\left\|\phi_{2}(s)\right\| \Delta s \int_{r_{1}}^{r_{2}} \frac{K_{0}}{1+\mu_{\varepsilon}(t)(\ominus \omega)} e_{\ominus \omega}(\sigma(t), \sigma(s)) \Delta t \\
& \leqslant \frac{1}{m\left(r_{1}, r_{2}, \tilde{\rho}\right)} K_{0}(1+(\bar{\mu}+\varepsilon) \omega) \int_{-\infty}^{r_{1}}\left\|\phi_{2}(s)\right\| \Delta s \int_{r_{1}}^{r_{2}} e_{\omega}(\sigma(s), \sigma(t)) \Delta t \\
& =\frac{1}{m\left(r_{1}, r_{2}, \tilde{\rho}\right)} \frac{K_{0}(1+(\bar{\mu}+\varepsilon) \omega)}{\omega} \int_{-\infty}^{r_{1}}\left\|\phi_{2}(s)\right\|\left[e_{\omega}\left(\sigma(s), r_{1}\right)-e_{\omega}\left(\sigma(s), r_{2}\right)\right] \Delta s \\
& \leqslant \frac{1}{m\left(r_{1}, r_{2}, \tilde{\rho}\right)} \frac{K_{0}(1+(\bar{\mu}+\varepsilon) \omega)}{\omega}\left\|\phi_{2}\right\|_{\varepsilon}\left(\int_{-\infty}^{r_{1}} e_{\ominus \omega}\left(r_{1}, \sigma(s)\right) \Delta s-\int_{-\infty}^{r_{1}} e_{\ominus \omega}\left(r_{2}, \sigma(s)\right) \Delta s\right)
\end{aligned}
$$




$$
\begin{aligned}
= & \frac{1}{m\left(r_{1}, r_{2}, \tilde{\rho}\right)} \frac{K_{0}(1+(\bar{\mu}+\varepsilon) \omega)}{\omega} \frac{\left\|\phi_{2}\right\|_{\varepsilon}}{\ominus \omega}\left(e_{\ominus \omega}\left(r_{1},-\infty\right)-e_{\ominus \omega}\left(r_{1}, r_{1}\right)\right. \\
& \left.-e_{\ominus \omega}\left(r_{2},-\infty\right)+e_{\ominus \omega}\left(r_{2}, r_{1}\right)\right) \rightarrow 0 \text { as } r_{2}-r_{1}:=D_{r} \rightarrow \infty,
\end{aligned}
$$

and

$$
\begin{aligned}
I_{2}^{0} & =\frac{1}{m\left(r_{1}, r_{2}, \tilde{\rho}\right)} \int_{r_{1}}^{r_{2}}\left\|\phi_{2}(s)\right\| \Delta s \int_{s}^{r_{2}} K_{0} e_{\ominus \omega}(t, \sigma(s)) \Delta t \\
& =\frac{1}{m\left(r_{1}, r_{2}, \tilde{\rho}\right)} \int_{r_{1}}^{r_{2}}\left\|\phi_{2}(s)\right\| \Delta s \int_{s}^{r_{2}} \frac{1}{1+\mu_{\varepsilon}(t)(\ominus \omega)} e_{\ominus \omega}(\sigma(t), \sigma(s)) \Delta t \\
& \leqslant \frac{1}{m\left(r_{1}, r_{2}, \tilde{\rho}\right)} K_{0}(1+(\bar{\mu}+\varepsilon) \omega) \int_{r_{1}}^{r_{2}}\left\|\phi_{2}(s)\right\| \Delta s \int_{s}^{r_{2}} e_{\omega}(\sigma(s), \sigma(t)) \Delta t \\
& =\frac{1}{m\left(r_{1}, r_{2}, \tilde{\rho}\right)} \frac{K_{0}(1+(\bar{\mu}+\varepsilon) \omega)}{\omega} \int_{r_{1}}^{r_{2}}\left\|\phi_{2}(s)\right\|\left[e_{\omega}(\sigma(s), s)-e_{\omega}\left(\sigma(s), r_{2}\right)\right] \Delta s \\
& \leqslant \frac{1}{m\left(r_{1}, r_{2}, \tilde{\rho}\right)} \frac{K_{0}(1+(\bar{\mu}+\varepsilon) \omega)^{2}}{\omega} \int_{r_{1}}^{r_{2}}\left\|\phi_{2}(s)\right\| \Delta s .
\end{aligned}
$$

Since $\phi_{2} \in \operatorname{WPDAP}_{0}^{\varepsilon}(\mathbb{T}, \rho)$, we find that $\lim _{\mathrm{D}_{\mathrm{r}} \rightarrow \infty} \frac{1}{\mathrm{~m}\left(\mathrm{r}_{1}, \mathrm{r}_{2}, \tilde{\rho}\right)} \int_{r_{1}}^{r_{2}}\left\|\phi_{2}(s)\right\| \Delta s=0$. Hence, $\lim _{\mathrm{D}_{\mathrm{r}} \rightarrow \infty} \mathrm{I}_{2}^{0}=0$.

It remains to show that $\Upsilon_{2} \in \operatorname{WPDAP}_{0}^{\varepsilon}(\mathbb{T}, \tilde{\rho})$. For any $D_{r}>0$, there exist $i(r), j(r)$ such that

$$
t_{i(r)-1}<r_{1} \leqslant t_{i(r)}<\ldots<t_{j(r)} \leqslant r_{2}<t_{j(r)+1} .
$$

Since $\gamma_{i} \in \operatorname{WPDAP}_{0}^{\varepsilon}(\mathbb{Z}, \rho), M_{\gamma_{i}}=\sup _{i \in \mathbb{Z}}\left\|\gamma_{i}\right\|<\infty$, and from the fact that for $a \in \mathscr{R}_{\mathbb{T}}(\tau, \varepsilon), e_{\ominus \omega}(t, a)=$ $\left(1+\mu_{\varepsilon}(t) \omega\right) e_{\omega}(a, \sigma(t))$, we find

$$
\begin{aligned}
\frac{1}{m\left(r_{1}, r_{2}, \tilde{\rho}\right)} \int_{r_{1}}^{r_{2}}\left\|r_{2}(t)\right\| \Delta t= & \frac{1}{m\left(r_{1}, r_{2}, \tilde{\rho}\right)} \int_{r_{1}}^{r_{2}}\left\|\sum_{t_{k_{i}, \varepsilon}<t} T\left(t, t_{k_{i}, \varepsilon}\right) \gamma_{i}\right\| \Delta t \\
\leqslant & \frac{1}{m\left(r_{1}, r_{2}, \tilde{\rho}\right)} \int_{r_{1}}^{r_{2}} \sum_{t_{k_{i}, \varepsilon}<t} K_{0} e_{\ominus \omega}\left(t, t_{k_{i}, \varepsilon}\right)\left\|\gamma_{i}\right\| \Delta t \\
\leqslant & \frac{1}{m\left(r_{1}, r_{2}, \tilde{\rho}\right)} \sum_{t_{k_{i}, \varepsilon}<r_{1}} K_{0} e_{\ominus \omega}\left(r_{1}, t_{k_{i}, \varepsilon}\right)\left\|\gamma_{i}\right\| \int_{r_{1}}^{r_{2}} e_{\ominus \omega}\left(t, r_{1}\right) \Delta t \\
& +\frac{1}{m\left(r_{1}, r_{2}, \tilde{\rho}\right)} \sum_{r_{1}<t_{k_{i}, \varepsilon}<r_{2}}\left\|\gamma_{i}\right\| \int_{r_{1}}^{r_{2}} K_{0} e_{\ominus \omega}\left(t, t_{k_{i}, \varepsilon}\right) \Delta t \\
\leqslant & \frac{1}{m\left(r_{1}, r_{2}, \tilde{\rho}\right)} \sum_{t_{k_{i}, \varepsilon}<r_{1}} \frac{K_{0}(1+(\bar{\mu}+\varepsilon) \omega)}{\omega} M_{\gamma_{i}} e_{\ominus \omega}\left(r_{1}, t_{k_{i}, \varepsilon}\right) \\
& +\frac{1}{m\left(r_{1}, r_{2}, \tilde{\rho}\right)} \sum_{r_{1}<t_{k_{i}, \varepsilon}<r_{2}} \frac{K_{0}(1+(\bar{\mu}+\varepsilon) \omega)}{\omega}\left\|\gamma_{i}\right\| \\
\leqslant & \frac{1}{m\left(r_{1}, r_{2}, \tilde{\rho}\right)} \frac{K_{0} M_{\gamma_{i}}(1+(\bar{\mu}+\varepsilon) \omega)}{\omega} \frac{1}{1-e_{\ominus \omega}\left(\theta_{\varepsilon}, 0\right)} \\
& +\frac{K_{0}(1+(\bar{\mu}+\varepsilon) \omega)}{\omega} \frac{1}{m\left(r_{1}, r_{2}, \tilde{\rho}\right)} \sum_{k=i(r)}^{j(r)}\left\|\gamma_{k}\right\| .
\end{aligned}
$$

Since $\gamma_{i} \in \operatorname{WPDAP}_{0}^{\varepsilon}(\mathbb{Z}, \tilde{\rho})$, and for $\mathrm{D}_{r} \rightarrow \infty, \mathrm{m}\left(\mathrm{r}_{1}, \mathrm{r}_{2}, \tilde{\rho}\right) \rightarrow \infty$, we have

$$
\lim _{D_{r} \rightarrow \infty} \frac{1}{m\left(r_{1}, r_{2}, \tilde{\rho}\right)} \sum_{k=i(r)}^{j(r)}\left\|\gamma_{k}\right\|=\lim _{D_{r} \rightarrow \infty} \frac{1}{\sum_{k=i(r)}^{j(r)} \tilde{\rho}\left(t_{k}\right) \mu\left(t_{k}\right)} \sum_{k=i(r)}^{j(r)}\left\|\gamma_{k}\right\|=0 .
$$


Further, as $D_{r} \rightarrow \infty$, one has

$$
\frac{1}{m\left(r_{1}, r_{2}, \tilde{\rho}\right)} \frac{K_{0} M_{\gamma_{i}}(1+(\bar{\mu}+\varepsilon) \omega)}{\omega} \frac{1}{1-e_{\ominus \omega}\left(\theta_{\varepsilon}, 0\right)} \rightarrow 0
$$

Hence, it follows that

$$
\lim _{D_{r} \rightarrow \infty} \frac{1}{m\left(r_{1}, r_{2}, \tilde{\rho}\right)} \int_{r_{1}}^{r_{2}}\left\|r_{2}(t)\right\| \Delta t=0
$$

Thus, $\sum_{t_{k_{i}, \varepsilon}<\cdot} T\left(\cdot, t_{k_{i}, \varepsilon}\right) I_{k}\left(\vartheta\left(t_{k_{i}, \varepsilon}\right)\right) \in \operatorname{WPDAP}_{0}^{\varepsilon}(\mathbb{T}, \tilde{\rho})$, and hence $F(\cdot) \in \operatorname{WPDAP}^{\varepsilon}(\mathbb{T}, \tilde{\rho})$. This completes the proof.

The first existence result is based on the Schauder fixed point theorem.

Theorem 3.15. Suppose conditions $\left(\mathrm{H}_{1}\right)-\left(\mathrm{H}_{4}\right)$ hold. Then Eq. (1.1) has a weighted pseudo double-almost periodic solution.

Proof. Let $\mathbb{D}=\left\{\varphi \in \operatorname{WPDAP}^{\varepsilon}(\mathbb{T}, \mathbb{X}) \cap \operatorname{UPC}^{\varepsilon}(\mathbb{T}, \mathbb{X}):\|\varphi\|_{\varepsilon} \leqslant \mathrm{L}_{0}\right\}$. Define an operator $\Gamma$ on $\mathbb{D}$ by

$$
\Gamma \varphi=\int_{-\infty}^{t} \mathrm{~T}(\mathrm{t}, \sigma(\mathrm{s})) \mathrm{f}(\mathrm{s}, \varphi(\mathrm{s})) \Delta \mathrm{s}+\sum_{\mathrm{t}_{\mathrm{k}_{i}, \varepsilon}<\mathrm{t}} \mathrm{T}\left(\mathrm{t}, \mathrm{t}_{\mathrm{k}_{\mathrm{i}}, \varepsilon}\right) \mathrm{I}_{\mathrm{k}}\left(\varphi\left(\mathrm{t}_{\mathrm{k}_{\mathrm{i}}, \varepsilon}\right)\right) .
$$

We will show that $\Gamma$ has a fixed point in $\mathbb{D}$. From Theorem 3.14, it follows that for every $\varphi \in \mathbb{D}$, $\Gamma \varphi \in \operatorname{WPDAP}^{\varepsilon}(\mathbb{T}, \rho)$.

Step 1. We will show that $\|\Gamma \varphi\|_{\varepsilon} \leqslant \mathrm{L}_{0}$. For every $\varphi \in \mathbb{D}$, by $\left(\mathrm{H}_{1}\right)$ and $\left(\mathrm{H}_{4}\right)$, we have

$$
\begin{aligned}
\|\Gamma \varphi(t)\| & =\left\|\int_{-\infty}^{t} T(t, \sigma(s)) f(s, \varphi(s)) \Delta s+\sum_{t_{k_{i}, \varepsilon}<t} T\left(t, t_{k_{i}, \varepsilon}\right) I_{k}\left(\varphi\left(t_{k_{i}, \varepsilon}\right)\right)\right\| \\
& \leqslant \int_{-\infty}^{t} K_{0} e_{\ominus \omega}(t, \sigma(s))\|f(s, \varphi(s))\| \Delta s+\sum_{t_{k_{i}, \varepsilon}<t} K_{0} e_{\ominus \omega}\left(t, t_{k_{i}, \varepsilon}\right)\left\|I_{k}\left(\varphi\left(t_{k_{i}, \varepsilon}\right)\right)\right\| \\
& \leqslant H_{1 L_{0}} K_{0} \int_{-\infty}^{t} e_{\ominus \omega}(t, \sigma(s)) \Delta s+H_{2 L_{0}} K_{0} \sum_{t_{k_{i}, \varepsilon}<t} e_{\ominus \omega}\left(t, t_{k_{i}, \varepsilon}\right) \\
& \leqslant \frac{\mathrm{H}_{1 L_{0}} K_{0}(1+(\bar{\mu}+\varepsilon) \omega)}{\omega} H_{1 L_{0}}+\frac{\mathrm{H}_{2 \mathrm{~L}_{0}} \mathrm{~K}_{0}}{1-e_{\ominus \omega}\left(\theta_{\varepsilon}, 0\right)} \leqslant \mathrm{L}_{0},
\end{aligned}
$$

thus $\|\Gamma \varphi\|_{\varepsilon} \leqslant \mathrm{L}_{0}$.

Step 2. We will show that for every $\varphi \in \mathbb{D}, \Gamma \varphi \in \operatorname{UPC}^{\varepsilon}(\mathbb{T}, \mathbb{X})$.

Suppose $\varphi \in \mathbb{D}, t^{\prime}, t^{\prime \prime} \in\left(t_{k_{i}, \varepsilon}, t_{k_{i+1}, \varepsilon}\right)_{\mathscr{R}_{\mathbb{T}}}(\tau, \varepsilon), i \in \mathbb{Z}$, by conditions (1) and (3) in Definition 3.8, we can easily see that for any $\varepsilon^{*}>\varepsilon>0$, there exists $\delta_{0}>0$ such that $0<t^{\prime}-t^{\prime \prime}<\delta_{0}=\frac{\varepsilon^{*} \omega}{3 K_{0}(1+(\bar{\mu}+\varepsilon) \omega) H_{1 L_{0}}}$ implies

$$
\left\|T\left(t^{\prime}, t^{\prime \prime}\right)-I\right\|<\min \left\{\frac{\varepsilon^{*} \omega}{3 K_{0}(1+(\bar{\mu}+\varepsilon) \omega) \mathrm{H}_{1 L_{0}}}, \frac{\varepsilon^{*}\left(1-e_{\ominus \omega}\left(\theta_{\varepsilon}, 0\right)\right)}{3 \mathrm{H}_{2} \mathrm{~L}_{0} \mathrm{~K}_{0}}\right\} .
$$

Thus, we have

$$
\begin{aligned}
\left\|\Gamma \varphi\left(t^{\prime}\right)-\Gamma \varphi\left(t^{\prime \prime}\right)\right\|= & \| \int_{-\infty}^{t^{\prime}} T\left(t^{\prime}, \sigma(s)\right) f(s, \varphi(s)) \Delta s-\int_{-\infty}^{t^{\prime \prime}} T\left(t^{\prime \prime}, \sigma(s)\right) f(s, \varphi(s)) \Delta s \\
& +\sum_{t_{k_{i}, \varepsilon}<t^{\prime}} T\left(t^{\prime}, s\right) I_{k}\left(\varphi\left(t_{k_{i}, \varepsilon}\right)\right)-\sum_{t_{k_{i}, \varepsilon}<t^{\prime \prime}} T\left(t^{\prime \prime}, t_{k_{i}, \varepsilon}\right) I_{k}\left(\varphi\left(t_{k_{i}, \varepsilon}\right)\right) \|
\end{aligned}
$$




$$
\begin{aligned}
& \leqslant\left\|\int_{-\infty}^{t^{\prime \prime}}\left[T\left(t^{\prime}, \sigma(s)\right)-T\left(t^{\prime \prime}, \sigma(s)\right)\right] f(s, \varphi(s)) \Delta s\right\|+\left\|\int_{t^{\prime \prime}}^{t^{\prime}} T\left(t^{\prime}, \sigma(s)\right) f(s, \varphi(s)) \Delta s\right\| \\
& +\left\|\sum_{t_{k_{i}, \varepsilon}<t^{\prime \prime}}\left[T\left(t^{\prime}, t_{k_{i}, \varepsilon}\right)-T\left(t^{\prime \prime}, t_{k_{i}, \varepsilon}\right)\right] I_{k}\left(\varphi\left(t_{k_{i}, \varepsilon}\right)\right)\right\| \\
& \leqslant \int_{-\infty}^{t^{\prime \prime}}\left\|\mathrm{T}\left(\mathrm{t}^{\prime \prime}, \sigma(s)\right)\right\|\left\|\mathrm{T}\left(\mathrm{t}^{\prime}, \mathrm{t}^{\prime \prime}\right)-\mathrm{I}\right\|\|\mathrm{f}(\mathrm{s}, \varphi(\mathrm{s}))\| \Delta s+\int_{\mathrm{t}^{\prime \prime}}^{\mathrm{t}^{\prime}}\left\|\mathrm{T}\left(\mathrm{t}^{\prime}, \sigma(s)\right)\right\|\|f(s, \varphi(s))\| \Delta s
\end{aligned}
$$

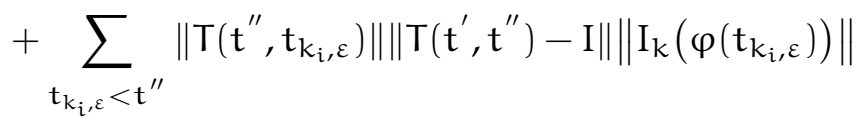

$$
\begin{aligned}
& \leqslant \mathrm{K}_{0} \mathrm{H}_{1 \mathrm{~L}_{0}} \frac{\varepsilon^{*} \omega}{3 \mathrm{~K}_{0}(1+(\bar{\mu}+\varepsilon) \omega) \mathrm{H}_{1 \mathrm{~L}_{0}}} \int_{-\infty}^{\mathrm{t}^{\prime \prime}} \mathrm{e}_{\ominus \omega}\left(\mathrm{t}^{\prime \prime}, \sigma(\mathrm{s})\right) \Delta \mathrm{s} \\
& +\mathrm{H}_{1 \mathrm{~L}_{0}} \mathrm{~K}_{0} \frac{1+(\bar{\mu}+\varepsilon) \omega}{\omega} \frac{\varepsilon^{*} \omega}{3 \mathrm{H}_{1 \mathrm{~L}_{0}} \mathrm{~K}_{0}(1+(\bar{\mu}+\varepsilon) \omega)} \\
& +\mathrm{H}_{2 \mathrm{~L}_{0}} \mathrm{~K}_{0} \frac{\varepsilon^{*}\left(1-e_{\ominus \omega}\left(\theta_{\varepsilon}, 0\right)\right)}{3 \mathrm{H}_{2 \mathrm{~L}_{0}} \mathrm{~K}_{0}} \sum_{\mathrm{t}_{\mathrm{k}_{\mathrm{i}}, \varepsilon}<\mathrm{t}^{\prime \prime}} e_{\ominus \omega}\left(\mathrm{t}^{\prime \prime}, \mathrm{t}_{\mathrm{k}_{\mathrm{i}}, \varepsilon}\right)<\frac{\varepsilon^{*}}{3}+\frac{\varepsilon^{*}}{3}+\frac{\varepsilon^{*}}{3}=\varepsilon^{*},
\end{aligned}
$$

which shows that $\Gamma \varphi \in \operatorname{UPC}^{\varepsilon}(\mathbb{T}, \mathbb{X})$. Hence, $\Gamma \mathbb{D} \subseteq \mathbb{D}$.

Step 3. We will show that $\Gamma$ is continuous.

Let $\left\{\varphi_{\mathrm{n}}\right\} \subset \mathbb{D} \subset \mathrm{PC}_{\mathrm{rd}}^{\varepsilon}(\mathbb{T}, \mathbb{X}), \varphi_{\mathrm{n}} \rightarrow \varphi$ in $\mathrm{PC}_{\mathrm{rd}}^{\varepsilon}(\mathbb{T}, \mathbb{X})$ as $\mathrm{n} \rightarrow \infty$. Then, we can find a bounded subset $\mathrm{K} \subseteq \Omega$ such that $\varphi\left(\mathscr{R}_{\mathbb{T}}(\tau, \varepsilon)\right) \subseteq \mathrm{K}, \mathrm{n} \in \mathbb{N}$. By $\left(\mathrm{H}_{2}\right)$, for any $\varepsilon^{*}>0$, there exists $\delta_{\mathcal{\varepsilon}^{*}}^{\prime}>0$ such that $x, y \in \mathrm{K}$ and $\|x-y\|<\delta_{\mathcal{E}^{*}}^{\prime}$ implies that

$$
\|f(t, x)-f(t, y)\|<\varepsilon^{*} \text { for all } t \in \mathscr{R}_{\mathbb{T}}(\tau, \varepsilon),
$$

and

$$
\left\|\mathrm{I}_{k}(\mathrm{x})-\mathrm{I}_{\mathrm{k}}(\mathrm{y})\right\|<\varepsilon^{*} \text { for all } k \in \mathbb{Z} .
$$

For the above $\delta_{\mathcal{\varepsilon}^{*}}^{\prime}$, there exists $\mathrm{n}_{0}$ such that $\left\|\varphi_{\mathrm{n}}(\mathrm{t})-\varphi(\mathrm{t})\right\|<\delta_{\mathcal{\varepsilon}^{*}}^{\prime}$ for $\mathrm{n}>\mathrm{n}_{0}$ and $\mathrm{t} \in \mathscr{R}_{\mathbb{T}}(\tau, \varepsilon)$, thus for $n>n_{0}$, we have

$$
\left\|f\left(t, \varphi_{n}(t)\right)-f(t, \varphi(t))\right\|<\varepsilon^{*} \text { for all } t \in \mathscr{R}_{\mathbb{T}}(\tau, \varepsilon),
$$

and

$$
\left\|\mathrm{I}_{k}\left(\varphi_{\mathrm{n}}\left(\mathrm{t}_{\mathrm{k}_{\mathrm{i}}, \varepsilon}\right)\right)-\mathrm{I}_{k}\left(\varphi\left(\mathrm{t}_{\mathrm{k}_{\mathrm{i}, \varepsilon}}\right)\right)\right\|<\varepsilon^{*} \text { for all } i \in \mathbb{Z} \text {. }
$$

Hence,

$$
\begin{aligned}
\left\|\Gamma \varphi_{n}(t)-\Gamma \varphi(t)\right\|= & \| \int_{-\infty}^{t} T(t, \sigma(s))\left[f\left(s, \varphi_{n}(s)\right)-f(s, \varphi(s))\right] \Delta s \\
& +\sum_{t_{k_{i}, \varepsilon}<t} T\left(t, t_{k_{i}, \varepsilon}\right)\left[I_{k}\left(\varphi_{n}\left(t_{k_{i}, \varepsilon}\right)\right)-I_{k}\left(\varphi\left(t_{k_{i}, \varepsilon}\right)\right)\right] \| \\
\leqslant & \int_{-\infty}^{t} K_{0} e_{\ominus \omega}(t, \sigma(s))\left\|f\left(s, \varphi_{n}(s)\right)-f(s, \varphi(s))\right\| \Delta s \\
& +\sum_{t_{k_{i}, \varepsilon}<t} K_{0} e_{\ominus \omega}\left(t, t_{k_{i}, \varepsilon}\right)\left\|I_{k}\left(\varphi_{n}\left(t_{k_{i}, \varepsilon}\right)\right)-I_{k}\left(\varphi\left(t_{k_{i}, \varepsilon}\right)\right)\right\| \\
\leqslant & {\left[\frac{K_{0}(1+(\bar{\mu}+\varepsilon) \omega)}{\omega}+\frac{K_{0}}{1-e_{\ominus \omega}\left(\theta_{\varepsilon}, 0\right)}\right] \varepsilon^{*}, }
\end{aligned}
$$

from which it follows that $\Gamma$ is continuous. 
Step 4. We will show that $\mathbb{D}_{\mathrm{t}}=\{\Gamma \varphi(\mathrm{t}): \varphi \in \mathbb{D}\}$ is a relatively compact subset of $\mathbb{X}$ for each $\mathrm{t} \in \mathscr{R}_{\mathbb{T}}(\tau, \varepsilon)$.

By condition (3) in Definition 3.8, for any $\varepsilon^{*}>\varepsilon>0$, there exists $\eta_{\varepsilon^{*}}>0$, such that $|t-s|<\eta_{\varepsilon^{*}}$ implies $\|T(t, s)-I\|_{B(\mathbb{X})}<\varepsilon^{*}$. Hence, we find that for $0<|t-s|<\eta_{\varepsilon^{*}}, T(t, s)$ is a bounded linear operator in $\mathbb{X}$. From $\left(H_{3}\right)$, i.e., $\mathbb{X}$ is a finite dimensional Banach space, $T(t, s)$ is compact. Let $\|T(t, s)\|_{B(\mathbb{X})} \leqslant N_{0}$ for $0<\mathrm{t}-\mathrm{t}_{\mathrm{c}}<\eta_{\varepsilon^{*}}$, where

$$
\eta_{\varepsilon^{*}}=\min \left\{\frac{\omega \varepsilon^{*}}{2 \mathrm{~N}_{0} \mathrm{~K}_{0} \mathrm{H}_{1 \mathrm{~L}_{0}}(1+(\bar{\mu}+\varepsilon) \omega)}, \frac{\left(1-e_{\ominus \omega}\left(\theta_{\varepsilon}, 0\right)\right) \varepsilon^{*}}{2 \mathrm{~N}_{0} \mathrm{~K}_{0} \mathrm{C}_{2 \mathrm{~L}_{0}}}\right\}
$$

For $\mathrm{t} \in \mathscr{R}_{\mathbb{T}}(\tau, \varepsilon)$, we define

$$
\begin{aligned}
\Gamma_{t_{c}} \varphi(t) & =\int_{-\infty}^{t_{c}} T(t, \sigma(s)) f(s, \varphi(s)) \Delta s+\sum_{t_{k_{i}, \varepsilon}<t_{c}} T\left(t, t_{k_{i}, \varepsilon}\right) I_{k}\left(\varphi\left(t_{k_{i}, \varepsilon}\right)\right) \\
& =T\left(t, t_{c}\right)\left[\int_{-\infty}^{t_{c}} T\left(t_{c}, \sigma(s)\right) f(s, \varphi(s)) \Delta s+\sum_{t_{k_{i}, \varepsilon}<t_{c}} T\left(t_{c}, t_{k_{i}, \varepsilon}\right) I_{k}\left(\varphi\left(t_{k_{i}, \varepsilon}\right)\right)\right]=T\left(t, t_{c}\right) \Gamma \varphi\left(t_{c}\right),
\end{aligned}
$$

and then we have

$$
\begin{aligned}
\Gamma \varphi(t)-\Gamma_{t_{c}} \varphi(t)= & \int_{-\infty}^{t} T(t, \sigma(s)) f(s, \varphi(s)) \Delta s+\sum_{t_{k_{i}, \varepsilon}<t} T\left(t, t_{k_{i}, \varepsilon}\right) I_{k}\left(\varphi\left(t_{k_{i}, \varepsilon}\right)\right) \\
& -\int_{-\infty}^{t_{c}} T(t, \sigma(s)) f(s, \varphi(s)) \Delta s+\sum_{t_{k_{i}, \varepsilon}<t_{c}} T\left(t, t_{k_{i}, \varepsilon}\right) I_{k}\left(\varphi\left(t_{k_{i}, \varepsilon}\right)\right) \\
= & T\left(t, t_{c}\right)\left[\int_{-\infty}^{t_{c}} T\left(t_{c}, \sigma(s)\right) f(s, \varphi(s)) \Delta s+\sum_{t_{k_{i}, \varepsilon}<t_{c}} T\left(t_{c}, t_{k_{i}, \varepsilon}\right) I_{k}\left(\varphi\left(t_{k_{i}, \varepsilon}\right)\right)\right. \\
& +\int_{t_{c}}^{t} T(t, \sigma(s)) f(s, \varphi(s)) \Delta s+\sum_{t_{c}<t} T\left(t, t_{k_{i}, \varepsilon}\right) I_{k}\left(\varphi\left(t_{k_{i}, \varepsilon}\right)\right) \\
& \left.-\int_{-\infty}^{t_{c}} T\left(t_{c}, \sigma(s)\right) f(s, \varphi(s)) \Delta s+\sum_{t_{k_{i}, \varepsilon}<t_{c}} T\left(t_{c}, t_{k_{i}, \varepsilon}\right) I_{k}\left(\varphi\left(t_{k_{i}, \varepsilon}\right)\right)\right] \\
= & T\left(t, t_{c}\right) \Gamma_{0} \varphi(t),
\end{aligned}
$$

where $\Gamma_{0} \varphi(t)=\int_{t_{c}}^{t} T(t, \sigma(s)) f(s, \varphi(s)) \Delta s+\sum_{t_{c}<t} T\left(t, t_{k_{i}, \varepsilon}\right) I_{k}\left(\varphi\left(t_{k_{i}, \varepsilon}\right)\right)$. Since $\left\{\Gamma \varphi\left(t_{c}\right): \varphi \in \mathbb{D}\right\}$ is bounded and $T\left(t, t_{c}\right)$ is compact, $\left\{T\left(t, t_{c}\right) \Gamma \varphi\left(t_{c}\right): \varphi \in \mathbb{D}\right\}$ is a relatively compact subset of $\mathbb{X}$.

Further, we have

$$
\begin{aligned}
\left\|\Gamma \varphi(t)-\Gamma_{t_{c}} \varphi(t)\right\| & =\left\|\int_{t_{c}}^{t} T(t, \sigma(s)) f(s, \varphi(s)) \Delta s+\sum_{t_{c}<t} T\left(t, t_{k_{i}, \varepsilon}\right) I_{k}\left(\varphi\left(t_{k_{i}, \varepsilon}\right)\right)\right\|\left\|T\left(t, t_{c}\right)\right\|_{B(X)} \\
& \leqslant\left[\frac{K_{0} H_{1 L_{0}}(1+(\bar{\mu}+\varepsilon) \omega)}{\omega} \int_{t_{c}}^{t} e_{\ominus \omega}(t, \sigma(s)) \Delta s+K_{0} H_{2} L_{0} \sum_{t_{c}<t} e_{\ominus \omega}\left(t, t_{k_{i}, \varepsilon}\right)\right]\left\|T\left(t, t_{c}\right)\right\|_{B(\mathbb{X})} \\
& \leqslant\left[\frac{N_{0} K_{0} H_{1 L_{0}}(1+(\bar{\mu}+\varepsilon) \omega)}{\omega}+\frac{N_{0} K_{0} H_{2} L_{0}}{1-e_{\ominus \omega}\left(\theta_{\varepsilon}, 0\right)}\right] \eta<\frac{\varepsilon^{*}}{2}+\frac{\varepsilon^{*}}{2}=\varepsilon^{*} .
\end{aligned}
$$

Thus, $\mathbb{D}_{t}=\{\Gamma \varphi(t), \varphi \in \mathbb{D}\}$ is a relatively compact subset of $\mathbb{X}$ for each $t \in \mathscr{R}_{\mathbb{T}}(\tau, \varepsilon)$.

By Step 2, we find that $\{\Gamma \varphi: \varphi \in \mathbb{D}\}$ is equicontinuous at each interval $\left(t_{k_{i}, \varepsilon}, t_{k_{i+1}, \varepsilon}\right)_{\mathscr{R}_{\mathbb{T}}(\tau, \varepsilon)}, i \in \mathbb{Z}$. Further, since $\{\Gamma \varphi: \varphi \in \mathbb{D}\} \subset \mathrm{PC}_{\mathrm{h}_{0}}^{\mathcal{E}}(\mathbb{T}, \mathbb{X})$, it is a relatively compact set, and hence $\Gamma$ is a compact operator. Since $\mathbb{D}$ is a closed convex set, it follows from the Schauder fixed point theorem that $\Gamma$ has a fixed point $\varphi$ in $\mathbb{D}$. This fixed point $\varphi$ satisfies the integral equation

$$
\varphi(t)=\int_{-\infty}^{t} T(t, \sigma(s)) \Delta s+\sum_{t_{k_{i}, \varepsilon}<t} T\left(t, t_{k_{i}, \varepsilon}\right) I_{k}\left(\varphi\left(t_{k_{i}, \varepsilon}\right)\right)
$$


for all $\mathrm{t} \in \mathscr{R}_{\mathbb{T}}(\tau, \varepsilon)$. For fixed $\mathrm{t}^{*} \in \mathscr{R}_{\mathbb{T}}(\tau, \varepsilon), \mathrm{t}^{*} \neq \mathrm{t}_{\mathrm{k}_{\mathrm{i}}, \varepsilon}, i \in \mathbb{Z}$, we have

$$
\varphi\left(t^{*}\right)=\int_{-\infty}^{t^{*}} \mathrm{~T}\left(\mathrm{t}^{*}, \sigma(s)\right) f(s, \varphi(s)) \Delta s+\sum_{\mathrm{t}_{\mathrm{k}_{i}, \varepsilon}<\mathrm{t}^{*}} \mathrm{~T}\left(\mathrm{t}^{*}, \mathrm{t}_{\mathrm{k}_{\mathrm{i}}, \varepsilon}\right) \mathrm{I}_{\mathrm{k}}\left(\varphi\left(\mathrm{t}_{\mathrm{k}_{\mathrm{i}}, \varepsilon}\right)\right) .
$$

Since the family $\left\{A(t): t \in \mathscr{R}_{\mathbb{T}}(\tau, \varepsilon)\right\}$ of operators in $\mathbb{X}$ generates an exponentially stable evolution system $\left\{T(t, s): t \geqslant s, t, s \in \mathscr{R}_{\mathbb{T}}(\tau, \varepsilon)\right\}$, we find

$$
\begin{aligned}
\varphi(t)= & \int_{-\infty}^{t} T(t, \sigma(s)) f(s, \varphi(s)) \Delta s+\sum_{t_{k_{i}, \varepsilon}<t} T\left(t, t_{k_{i}, \varepsilon}\right) I_{k}\left(\varphi\left(t_{k_{i}, \varepsilon}\right)\right) \\
= & \int_{-\infty}^{t^{*}} T(t, \sigma(s)) f(s, \varphi(s)) \Delta s+\sum_{t_{k_{i}, \varepsilon}<t^{*}} T\left(t, t_{k_{i}, \varepsilon}\right) I_{k}\left(\varphi\left(t_{k_{i}, \varepsilon}\right)\right) \\
& +\int_{t^{*}}^{t} T(t, \sigma(s)) f(s, \varphi(s)) \Delta s+\sum_{t^{*}<t_{k_{i}, \varepsilon}<t} T\left(t, t_{k_{i}, \varepsilon}\right) I_{k}\left(\varphi\left(t_{k_{i}, \varepsilon}\right)\right) \\
= & T\left(t, t^{*}\right) \varphi\left(t^{*}\right)+\int_{t^{*}}^{t} T(t, \sigma(s)) f(s, \varphi(s)) \Delta s+\sum_{t^{*}<t_{k_{i}, \varepsilon}<t} T\left(t, t_{k_{i}, \varepsilon}\right) I_{k}\left(\varphi\left(t_{k_{i}, \varepsilon}\right)\right) .
\end{aligned}
$$

Therefore, $\varphi$ is a weighted piecewise pseudo double-almost periodic mild solution for Eq. (1.1) on an almost-complete closedness time scale $\mathbb{T}$. This completes the proof.

The following existence result is based on the contraction principle.

Theorem 3.16. Assume the following conditions hold:

$\left(A_{1}\right)$ The family $\left\{A(t): t \in \mathscr{R}_{\mathbb{T}}(\tau, \varepsilon)\right\}$ of operators in $\mathbb{X}$ generates an exponentially stable evolution system $\left\{\mathrm{T}(\mathrm{t}, \mathrm{s}): \mathrm{t} \geqslant \mathrm{s}, \mathrm{t}, \mathrm{s} \in \mathscr{R}_{\mathbb{T}}(\tau, \varepsilon)\right\}$, i.e., there exist $\mathrm{K}_{0}>1$ and $\omega>0$ such that

$$
\|T(t, s)\|_{B(\mathbb{X})} \leqslant K_{0} e_{\ominus \omega}(t, s), t \geqslant s,
$$

and for any $\varepsilon_{2}>\varepsilon_{1}>0$, there exists a relatively dense set $\overline{\mathrm{T}} \subset \Pi_{\varepsilon_{1}}$ such that if $\tau \in \overline{\mathrm{T}}$, then

$$
\|\mathrm{T}(\mathrm{t}+\tau, \mathrm{s}+\tau)-\mathrm{T}(\mathrm{t}, \mathrm{s})\|_{\mathrm{B}(\mathbb{X})}<\varepsilon_{2} e_{\ominus \omega}(\mathrm{t}, \mathrm{s}), \mathrm{t} \geqslant s, \mathrm{t}, \mathrm{s} \in \mathscr{R}_{\mathrm{T}}\left(\tau, \varepsilon_{1}\right) ;
$$

$\left(A_{2}\right) f \in \operatorname{WPDAP}^{\varepsilon}(\mathbb{T} \times \Omega, \tilde{\rho})$, and $f$ satisfies the Lipschitz condition with respect to the second argument, i.e.,

$$
\|f(t, x)-f(t, y)\| \leqslant L_{1}\|x-y\|, t \in \mathscr{R}_{\mathbb{T}}(\tau, \varepsilon), x, y \in \Omega ;
$$

$\left(A_{3}\right) I_{k}$ is a weighted pseudo-almost periodic sequence, and there exists a number $\mathrm{L}_{2}>0$ such that

$$
\left\|I_{k}(x)-I_{k}(y)\right\| \leqslant L_{2}\|x-y\|
$$

for all $x, y \in \Omega, k \in \mathbb{Z}$.

Further, assume that

$$
\frac{\mathrm{K}_{0} \mathrm{~L}_{1}(1+(\bar{\mu}+\varepsilon) \omega)}{\omega}+\frac{\mathrm{K}_{0} \mathrm{~L}_{2}}{1-e_{\ominus \omega}\left(\theta_{\varepsilon}, 0\right)}<1
$$

where $\bar{\mu}=\sup _{t \in \mathbb{T}} \mu$ and $e_{\ominus \omega}\left(\theta_{\varepsilon}, 0\right):=\sup _{i \in \mathbb{Z}} e_{\ominus \omega}\left(t_{k_{i+1}, \varepsilon}, t_{k_{i}, \varepsilon}\right)$. Then, Eq. (1.1) has a weighted pseudo double-almost periodic solution. 
Proof. Define the operator $\Gamma$ on $\operatorname{WPDAP}^{\varepsilon}(\mathbb{T}, \tilde{\rho}) \cap \operatorname{UPC}^{\varepsilon}(\mathbb{T}, \mathbb{X})$ as in the proof of Theorem 3.15. For $\varphi \in$ $\operatorname{WPDAP}^{\varepsilon}(\mathbb{T}, \tilde{\rho}) \cap \operatorname{UPC}^{\varepsilon}(\mathbb{T}, \mathbb{X})$, by $\left(A_{2}\right)$ and $\left(A_{3}\right)$, we have

$$
\|f(t, \varphi(t))\| \leqslant\|f(t, \varphi(t))-f(t, 0)\|+\|f(t, 0)\| \leqslant L_{1}\|\varphi(t)\|+\|f(t, 0)\|,
$$

and

$$
\left\|\mathrm{I}_{k}\left(\varphi\left(\mathrm{t}_{\mathrm{k}_{\mathrm{i}, \varepsilon}}\right)\right)\right\| \leqslant\left\|\mathrm{I}_{\mathrm{k}}\left(\varphi\left(\mathrm{t}_{\mathrm{k}_{\mathrm{i}, \varepsilon}}\right)\right)-\mathrm{I}_{\mathrm{i}}(0)\right\| \leqslant \mathrm{L}_{2}\left\|\varphi\left(\mathrm{t}_{\mathrm{k}_{\mathrm{i}, \varepsilon}}\right)\right\|+\left\|\mathrm{I}_{\mathrm{k}}(0)\right\| .
$$

Thus, $f(\cdot, \varphi(\cdot)) \in \operatorname{PC}_{r_{d}}^{\varepsilon}(\mathbb{T}, \mathbb{X})$ and $I_{k}\left(\varphi\left(t_{\left.k_{i}, \varepsilon\right)}\right)\right)$ is a bounded sequence. Therefore, it follows from the proof of Theorem 3.15 that $\Gamma \varphi \in \operatorname{WPDAP}^{\varepsilon}(\mathbb{T}, \tilde{\rho}) \cap \operatorname{UPC}^{\varepsilon}(\mathbb{T}, \mathbb{X})$. Hence, $\Gamma\left(\operatorname{WPDAP}^{\varepsilon}(\mathbb{T}, \tilde{\rho}) \cap \operatorname{UPC}^{\varepsilon}(\mathbb{T}, \mathbb{X})\right) \subset$ $\operatorname{WPDAP}^{\varepsilon}(\mathbb{T}, \tilde{\rho}) \cap \operatorname{UPC}^{\varepsilon}(\mathbb{T}, \mathbb{X})$. It suffices now to show that the operator $\Gamma$ has a fixed point in WPDAP ${ }^{\varepsilon}$ $(\mathbb{T}, \tilde{\rho}) \cap \operatorname{UPC}^{\varepsilon}(\mathbb{T}, \mathbb{X})$. For $\varphi_{1}, \varphi_{2} \in \operatorname{WPDAP}^{\varepsilon}(\mathbb{T}, \tilde{\rho}) \cap \operatorname{UPC}^{\varepsilon}(\mathbb{T}, \mathbb{X})$, we have

$$
\begin{aligned}
& \left\|\Gamma \varphi_{1}(\mathrm{t})-\Gamma \varphi_{2}(\mathrm{t})\right\|=\| \int_{-\infty}^{\mathrm{t}} \mathrm{T}(\mathrm{t}, \sigma(\mathrm{s}))\left[\mathrm{f}\left(\mathrm{s}, \varphi_{1}(\mathrm{~s})\right)-\mathrm{f}\left(\mathrm{s}, \varphi_{2}(\mathrm{~s})\right)\right] \Delta \mathrm{s} \\
& +\sum_{t_{k_{i}, \varepsilon}<t} T\left(t, t_{k_{i}, \varepsilon}\right)\left[I_{k}\left(\varphi_{1}\left(t_{k_{i}, \varepsilon}\right)\right)-I_{k}\left(\varphi_{2}\left(t_{k_{i}, \varepsilon}\right)\right)\right] \| \\
& \leqslant \int_{-\infty}^{t} K_{0} e_{\ominus \omega}(t, \sigma(s))\left\|f\left(s, \varphi_{1}(s)\right)-f\left(s, \varphi_{2}(s)\right)\right\| \Delta s \\
& +\sum_{t_{k_{i}, \varepsilon}<t} K_{0} e_{\ominus \omega}\left(t, t_{k_{i}, \varepsilon}\right)\left\|I_{k}\left(\varphi_{1}\left(t_{k_{i}, \varepsilon}\right)\right)-I_{k}\left(\varphi_{2}\left(t_{k_{i}, \varepsilon}\right)\right)\right\| \\
& \leqslant \int_{-\infty}^{t} \mathrm{~K}_{0} e_{\ominus \omega}(\mathrm{t}, \sigma(s)) \mathrm{L}_{1}\left\|\varphi_{1}(\mathrm{~s})-\varphi_{2}(\mathrm{~s})\right\| \Delta s \\
& +\sum_{t_{k_{i}, \varepsilon}<t} K_{0} e_{\ominus \omega}\left(t, t_{k_{i}, \varepsilon}\right) L_{2}\left\|\varphi_{1}\left(t_{k_{i}, \varepsilon}\right)-\varphi_{2}\left(t_{k_{i}, \varepsilon}\right)\right\| \\
& \leqslant\left[\frac{\mathrm{K}_{0} \mathrm{~L}_{1}(1+(\bar{\mu}+\varepsilon) \omega)}{\omega}+\frac{\mathrm{K}_{0} \mathrm{~L}_{2}}{1-e_{\ominus \omega}\left(\theta_{\varepsilon}, 0\right)}\right]\left\|\varphi_{1}-\varphi_{2}\right\| .
\end{aligned}
$$

Since $\frac{\mathrm{K}_{0} \mathrm{~L}_{1}(1+(\bar{\mu}+\varepsilon) \omega)}{\omega}+\frac{\mathrm{K}_{0} \mathrm{~L}_{2}}{1-e_{\ominus \omega}\left(\theta_{\varepsilon}, 0\right)}<1, \Gamma$ is a contradiction. Hence, $\Gamma$ has a fixed point in $\operatorname{WPDAP}^{\varepsilon}(\mathbb{T}, \tilde{\rho}) \cap \operatorname{UPC}^{\varepsilon}(\mathbb{T}, \mathbb{X})$. Thus Eq. (1.1) has a weighted pseudo double-almost periodic solution. This completes the proof.

Finally, we investigate the stability of a weighted pseudo double-almost periodic solution for Eq. (1.1).

Theorem 3.17. Suppose the conditions of Theorem 3.16 hold. Assume further that $(\ominus \omega) \oplus p<0$, where $p=$ $\mathrm{K}_{0} \mathrm{~L}_{1}(1+(\bar{\mu}+\varepsilon) \omega), \bar{\mu}=\sup _{\mathrm{t} \in \mathbb{T}} \mu(\mathrm{t})$. Then, Eq. (1.1) has an exponentially stable weighted pseudo double-almost periodic mild solution.

Proof. By Theorem 3.16, we know that Eq. (1.1) has a weighted piecewise pseudo double-almost periodic mild solution $u(t)$, and in integral form can be written as:

$$
u(t)=T\left(t, s_{0}\right) u\left(s_{0}\right)+\int_{s_{0}}^{t} T(t, \sigma(s)) f(s, u(s)) \Delta s+\sum_{s_{0}<t_{k_{i}, \varepsilon}<t} T\left(t, t_{k_{i}, \varepsilon}\right) I_{k}\left(u\left(t_{k_{i}, \varepsilon}\right)\right),
$$

where $t>s_{0}, s_{0} \neq t_{k_{i}, \varepsilon}, i \in \mathbb{Z}$.

Let $u(t)=u\left(t, s_{0}\right)$ and $v(t)=v\left(t, s_{0}\right)$ be two solutions of Eq. (1.1), then

$$
\begin{aligned}
& u(t)=T\left(t, s_{0}\right) u\left(s_{0}\right)+\int_{s_{0}}^{t} T(t, \sigma(s)) f(s, u(s)) \Delta s+\sum_{s_{0}<t_{k_{i}, \varepsilon}<t} T\left(t, t_{k_{i}, \varepsilon}\right) I_{k}\left(u\left(t_{k_{i}, \varepsilon}\right)\right), \\
& v(t)=T\left(t, s_{0}\right) v\left(s_{0}\right)+\int_{s_{0}}^{t} T(t, \sigma(s)) f(s, v(s)) \Delta s+\sum_{s_{0}<t_{k_{i}, \varepsilon}<t} T\left(t, t_{k_{i}, \varepsilon}\right) I_{k}\left(v\left(t_{k_{i}, \varepsilon}\right)\right) .
\end{aligned}
$$


Thus, it follows that

$$
\begin{aligned}
\|u(t)-v(t)\| \leqslant & \left\|T\left(t, s_{0}\right)\left[u\left(s_{0}\right)-v\left(s_{0}\right)\right]\right\|+\left\|\int_{s_{0}}^{t} T(t, \sigma(s))[f(s, u(s))-f(s, v(s))] \Delta s\right\| \\
& +\| \sum_{s_{0}<t_{k_{i}, \varepsilon}<t} T\left(t, t_{k_{i}, \varepsilon}\right)\left[\mathrm{I}_{k}\left(u\left(t_{k_{i}, \varepsilon}\right)\right)-I_{k}\left(v\left(t_{k_{i}, \varepsilon}\right)\right] \|\right. \\
\leqslant & \left\|T\left(t, s_{0}\right)\right\|\left\|u\left(s_{0}\right)-v\left(s_{0}\right)\right\|+\int_{s_{0}}^{t}\|T(t, \sigma(s))\|\|f(s, u(s))-f(s, v(s))\| \Delta s \\
& +\sum_{s_{0}<t_{k_{i}, \varepsilon}<t}\left\|T\left(t, t_{k_{i}, \varepsilon}\right)\right\|\left\|\left[\mathrm{I}_{k}\left(u\left(t_{k_{i}, \varepsilon}\right)\right)-I_{k}\left(v\left(t_{k_{i}, \varepsilon}\right)\right)\right]\right\| \\
\leqslant & K_{0} e_{\ominus \omega}\left(t, s_{0}\right)\left\|u\left(s_{0}\right)-v\left(s_{0}\right)\right\|+\int_{s_{0}}^{t} K_{0} L_{1} e_{\ominus \omega}(t, \sigma(s))\|u(s)-v(s)\| \Delta s \\
& +\sum_{s_{0}<t_{k_{i}, \varepsilon}<t} K_{0} L_{2} e_{\ominus \omega}\left(t, t_{k_{i}, \varepsilon}\right)\left\|u\left(t_{k_{i}, \varepsilon}\right)-v\left(t_{k_{i}, \varepsilon}\right)\right\| .
\end{aligned}
$$

Let $y(t)=\|u(t)-v(t)\| e_{\omega}\left(t, t_{0}\right)$, so that the above inequality can be written as

$$
y(t) \leqslant K_{0} y\left(s_{0}\right)+K_{0} L_{1}(1+(\bar{\mu}+\varepsilon) \omega) \int_{s_{0}}^{t} y(s) \Delta s+K_{0} L_{2} \sum_{s_{0}<t_{k_{i}, \varepsilon}<t} y\left(t_{k_{i}, \varepsilon}\right) .
$$

Now, from Gronwall-Bellman's inequality on time scales, we have

$$
y(t) \leqslant K_{0} y\left(s_{0}\right) \prod_{s_{0}<t_{k_{i}, \varepsilon}<t} K_{0} L_{2} e_{p}\left(t, t_{0}\right), t \in \mathscr{R}_{\mathbb{T}}(\tau, \varepsilon),
$$

which is the same as

$$
\|u(t)-v(t)\| \leqslant K_{0}\left\|u\left(s_{0}\right)-v\left(s_{0}\right)\right\| \prod_{s_{0}<t_{k_{i}, \varepsilon}<t} K_{0} L_{2} e_{p}\left(t, t_{0}\right) e_{(\ominus w) \oplus p}\left(t, t_{0}\right) .
$$

Hence, Eq. (1.1) has an exponentially stable weighted pseudo double-almost periodic mild solution. This completes the proof.

\section{Applications}

Here we propose some new dynamic models based on ACCTS in the real world, and some practical applications of our main results will be provided, which indicate that this theory works well, and not only unifies continuous and discrete situations, but also includes the situations "in between" in a broader sense. The models established on ACCTS are more suitable for precisely describing the reality with the law of almost cycle in practice.

The first application is to investigate the existence and stability of weighted pseudo double-almost periodic solutions of IBVP for a $\Delta$-partial dynamic equation.

Application 4.1. Let $\mathbb{T}$ be an almost-complete closedness time scale with $\bar{\mu}<\frac{5}{3}$ and $u \in \mathrm{PC}_{\mathrm{rd}}^{\varepsilon}(\mathbb{T} \times$ $\left.[0, \pi]_{\mathbb{T}}, \mathbb{R}\right)$. Consider the following impulsive partial dynamic equation:

$$
\left\{\begin{aligned}
& \frac{\partial}{\Delta_{1} t} u(t, x)= \frac{\partial^{2}}{\Delta_{2} x^{2}} u(t, x)+\frac{1}{180}(\sin t+\sin \sqrt{2} t+g(t)) \cos u(t, x), \\
& t \in\left(\mathscr{R}_{\mathbb{T}}(\tau, \varepsilon)\right) \backslash \cup \mathfrak{B}^{\varepsilon}, x \in\left[t_{k_{i_{1}, \varepsilon}}, t_{k_{i_{2}}, \varepsilon}\right]_{\mathscr{R}_{\mathbb{T}}(\tau, \varepsilon)}, i_{1}, i_{2} \in \mathbb{Z}, \\
& \Delta u\left(t_{k_{i}, \varepsilon}, x\right)=\beta_{k} u\left(t_{k_{i}, \varepsilon}, x\right), i \in \mathbb{Z}, x \in\left[t_{k_{i_{1}}, \varepsilon}, t_{k_{i_{2}}, \varepsilon}\right]_{\mathscr{R}_{\mathbb{T}}(\tau, \varepsilon)}, t_{k_{i_{i}, \varepsilon}} \in \cup \mathfrak{B}^{\varepsilon}, \\
& u\left(t, t_{k_{i_{1}}, \varepsilon}\right)=u\left(t, t_{k_{i_{2}}, \varepsilon}\right)=\varepsilon, t \in \mathscr{R}_{\mathbb{T}}(\tau, \varepsilon),
\end{aligned}\right.
$$


where $g \in \operatorname{UPC}^{\varepsilon}(\mathbb{T}, \mathbb{R})$ satisfies $|g(t)| \leqslant 1,\left(t \in \mathscr{R}_{\mathbb{T}}(\tau, \varepsilon)\right)$ and

$$
\begin{aligned}
\mathrm{t}_{\mathrm{k}_{\mathrm{i}_{1}}, \varepsilon} & =\inf \left\{\mathrm{t} \in \mathscr{R}_{\mathbb{T}}(\tau, \varepsilon): \mathrm{t}>0\right\}, & \mathrm{t}_{\mathrm{k}_{\mathrm{i}_{2}}, \varepsilon} & =\sup \left\{\mathrm{t} \in \mathscr{R}_{\mathbb{T}}(\tau, \varepsilon): \mathrm{t}<\pi\right\}, \\
\tilde{\rho}(\mathrm{t}) & =|\sin \mathrm{t}|+1, & \beta_{\mathrm{k}} & =\frac{1}{180}(\sin \mathrm{k}+\sin \sqrt{2} \mathrm{k}+\mathrm{g}(\mathrm{k})),
\end{aligned}
$$

and

$$
\mathfrak{B}^{\varepsilon}=\left\{t_{k_{i}, \varepsilon}=k_{i}+\frac{1}{8}\left|\sin k_{i}-\sin \sqrt{2} k_{i}\right|: i \in \mathbb{Z}\right\} .
$$

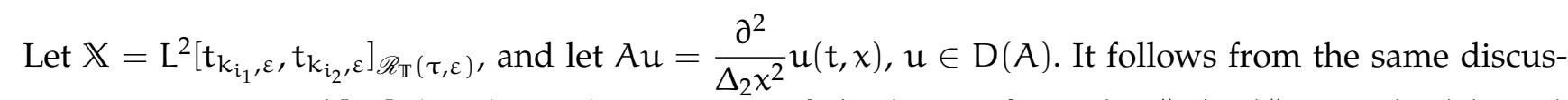
sion as in Section 3.1 of [19] that the evolution system $\{T(t, s): t \geqslant s\}$ satisfies $\|T(t, s)\| \leqslant e_{\ominus^{\frac{1}{2}}}(t, s)(t \geqslant s)$ with $K_{0}=1, w=1 / 2$. Further, it is easy to check that $\left\{t_{k_{i}, \varepsilon}^{j}\right\}, i, j \in \mathbb{Z}$ is an equipotentially almost periodic sequence on $\mathbb{R}$, and

$$
\begin{aligned}
t_{k}^{1}=t_{k+1}-t_{k} & =1+\frac{1}{8}|\sin (k+1)-\sin \sqrt{2}(k+1)|-\frac{1}{8}|\sin k-\sin \sqrt{2} k| \\
& \geqslant 1-\frac{1}{8}|\sin (k+1)-\sin k-[\sin \sqrt{2}(k+1)-\sin \sqrt{2} k]| \\
& \geqslant 1-\frac{1}{4}\left|\sin \frac{1}{2} \cos \frac{2 k+1}{2}\right|-\frac{1}{2}\left|\sin \frac{\sqrt{2}}{2} \cos \frac{\sqrt{2}(2 k+1)}{2}\right| \\
& \geqslant 1-\frac{1}{4} \sin \frac{1}{2}-\frac{1}{2} \sin \frac{\sqrt{2}}{2}>\frac{2}{5} .
\end{aligned}
$$

Hence, for any $\left\{s_{n}\right\} \subset \mathbb{Z}$, there exists a subsequence $\left\{s_{n_{m}}\right\}$ such that $\lim _{m \rightarrow \infty} t_{k_{i}, \varepsilon}^{s_{n_{m}}}$ uniformly exists for all $i \in \mathbb{Z}$, let

$$
\mathrm{I}_{0}=\left\{\mathfrak{i}_{0}: \mathrm{t}_{\mathrm{k}_{\mathrm{i}_{0}}, \varepsilon} \in \mathscr{R}_{\mathbb{T}}(\tau, \varepsilon)\right\},
$$

and one can obtain that for any $\left\{s_{n}\right\} \subset \mathbb{Z}$, there exists a subsequence $\left\{s_{n_{\mathfrak{m}}}\right\}$ such that $\lim _{m \rightarrow \infty} t_{k_{i_{0}}, \varepsilon}^{s_{n_{m}}}$ uniformly exists for all $i_{0} \in \mathrm{I}_{0}$. Thus, $\left\{\mathrm{t}_{\mathrm{k}_{\mathrm{i}_{0}}, \varepsilon}\right\} \in \mathfrak{B}^{\varepsilon}$ is an equipotentially almost periodic sequence and $\theta_{\varepsilon}=\inf _{i_{0} \in I_{0}}\left(t_{k_{i_{0}+1}, \varepsilon}-t_{k_{i_{0}}, \varepsilon}\right)>\frac{2}{5}+\varepsilon>0,\left(t_{k_{i_{0}}, \varepsilon} \in \cup \mathfrak{B}^{\varepsilon}\right)$. Now, let $f(t, u)=\hat{c} \frac{1}{180}(\sin t+\sin \sqrt{2} t+$ $g(t)) \cos u$, where $\hat{c}$ is some constant related to the number $\varepsilon$ satisfying $\hat{c}<1-e_{\ominus \omega}\left(\theta_{\varepsilon}, 0\right)$ and $I_{k}(u)=$ $\beta_{k} u=\frac{1}{60} \hat{c} u$.

Clearly, both $f$ and $I_{k}$ satisfy the assumptions of Theorems 3.16 and 3.17 with $L_{1}=L_{2}=\frac{1}{60} \hat{c}$. Moreover,

$$
\begin{aligned}
\frac{\mathrm{K}_{0} \mathrm{~L}_{1}(1+(\bar{\mu}+\varepsilon) \omega)}{\omega}+\frac{\mathrm{K}_{0} \mathrm{~L}_{2}}{1-e_{\ominus \omega}\left(\theta_{\varepsilon}, 0\right)} & <\frac{1}{30} \hat{\mathrm{c}}\left(\frac{11}{6}+\frac{\varepsilon}{2}\right)+\frac{\hat{\mathrm{c}}}{60\left(1-e_{\ominus \omega}\left(\theta_{\varepsilon}, 0\right)\right)} \\
& <\frac{1}{30}\left(\frac{11}{6}+\frac{\varepsilon}{2}\right)+\frac{1}{60} \\
& <\frac{1}{30}\left(\frac{11}{6}+\frac{1}{2}\right)+\frac{1}{60} \approx 0.0944<1 \text { for } \varepsilon<1,
\end{aligned}
$$

and since $\bar{\mu}<\frac{5}{3}$, it follows that $1+(\bar{\mu}+\varepsilon) \omega<\frac{11}{6}$, and hence

$$
\begin{aligned}
(\ominus \omega) \oplus p & =-\frac{\omega}{1+\mu_{\varepsilon} \omega}+K_{0} L_{1}(1+(\bar{\mu}+\varepsilon) \omega)-\frac{K_{0} L_{1} \omega \mu_{\varepsilon}(1+(\bar{\mu}+\varepsilon) \omega)}{\left(1+\mu_{\varepsilon} \omega\right)} \\
& \leqslant-\frac{\omega}{1+(\bar{\mu}+\varepsilon) \omega}+K_{0} L_{1}(1+(\bar{\mu}+\varepsilon) \omega)
\end{aligned}
$$




$$
<-0.2143+0.0389 \hat{\mathrm{c}}<-0.2143+0.0389<0 \text { for } \varepsilon<1 .
$$

Therefore, Eq. (4.1) has a weighted piecewise pseudo double-almost periodic mild solution which is exponentially stable.

Now, we apply our results to study dynamical models occurring in economics.

Application 4.2. A famous economic model is known as the Keynesian-Cross model that was earlier studied in [26]. However one sees that the classical Keynesian-Cross model has several deficiencies to describe aggregate income precisely in practice since several factors like the aggregate demand, the aggregate consumption, the aggregate investment and the government spending are not always almost the same after an exactly equivalent time interval, but they may always follow the law that they are almost the same after an almost equivalent time interval. Therefore, government is only able to strike a rough balance between supply and demand after an almost equivalent time interval rather than after the exactly equivalent time interval. Furthermore, some factors like the aggregate demand, the aggregate consumption, the aggregate investment and the government spending may suffer from sudden changes, for example, bankrupt of an enterprise will lead to a sudden decrease of personal income that will facilitate consumption level. Considering the almost periodicity of time variables and sudden changes of status of the model, we should optimize and fill the gaps of the classical Keynesian-Cross model. In the following, we consider the dynamic model in a simple closed economy with impulses on an almost-complete closedness time scale $\mathbb{T}$ :

$$
\begin{aligned}
\mathrm{D}(\mathrm{t}) & =\mathrm{C}(\mathrm{t})+\mathrm{I}_{0}(\mathrm{t}, \mathrm{y})+\mathrm{G}(\mathrm{t}, \mathrm{y}), \mathrm{t} \in\left(\mathscr{R}_{\mathbb{T}}(\tau, \varepsilon)\right) \backslash \cup \mathfrak{B}^{\varepsilon}, \\
\mathrm{C}(\mathrm{t}) & =\mathrm{C}_{0}(\mathrm{t}, \mathrm{y})+\mathrm{cy}(\mathrm{t}), \mathrm{t} \in\left(\mathscr{R}_{\mathbb{T}}(\tau, \varepsilon)\right) \backslash \cup \mathfrak{B}^{\varepsilon}, \\
\mathrm{y}^{\Delta}(\mathrm{t}) & =\delta\left[\mathrm{D}^{\sigma}-\mathrm{y}\right], \mathrm{t} \geqslant \mathrm{a}, \mathrm{t} \in\left(\mathscr{R}_{\mathbb{T}}(\tau, \varepsilon)\right) \backslash \cup \mathfrak{B}^{\varepsilon}, \mathrm{t} \neq \mathrm{t}_{\mathrm{k}_{i}, \varepsilon}, i \in \mathbb{Z}, \\
\Delta \mathrm{y}\left(\mathrm{t}_{\mathrm{k}_{i}, \varepsilon}\right) & =\mathrm{I}\left(\mathrm{y}\left(\mathrm{t}_{\mathrm{k}_{i}, \varepsilon}\right)\right)=\mathrm{y}\left(\mathrm{t}_{\mathrm{k}_{\mathrm{i}}, \varepsilon}^{+}\right)-\mathrm{y}\left(\mathrm{t}_{\mathrm{k}_{\mathrm{i}}, \varepsilon}^{-}\right), \mathrm{t}=\mathrm{t}_{\mathrm{k}_{\mathrm{i}, \varepsilon}} \in \cup \mathfrak{B}^{\varepsilon}, i \in \mathbb{Z},
\end{aligned}
$$

where $\mathrm{D}$ is the aggregate demand, $\mathrm{y}$ is the aggregate income, $\mathrm{C}$ is the aggregate consumption, $\mathrm{I}_{0}$ is the aggregate investment, $\mathrm{G}$ is the government spending, $\delta<1$ is a positive constant known as the speed of adjustment term, $\mathrm{C}_{0}$ is the additional consumer income, and $\mathrm{c}$ is non-negative constant.

In [26], $\mathrm{G}$ and $\mathrm{I}_{0}$ are taken as constants in (4.2), and current consumption is assumed to depend on current income in (4.3). Also, Eq. (4.4) means that the change in income is a fraction of excess demand at $\sigma(t)$ over income at $t$ (see [26]). However the aggregate investment $I_{0}$ and the government spending $G$ always depend on the aggregate income in real application. Hence, it is more appropriate to assume that $\mathrm{I}_{0}, \mathrm{G}, \mathrm{C}_{0}: \mathbb{T} \times \mathbb{R} \rightarrow \mathbb{R}$ are weighted pseudo double-almost periodic functions, which satisfy the followings:

$$
\begin{array}{r}
\left|I_{0}(t, x)-I_{0}(t, y)\right| \leqslant L_{1}|x-y|, \forall x, y \in \mathbb{R}, \\
|G(t, x)-G(t, y)| \leqslant L_{2}|x-y|, \forall x, y \in \mathbb{R}, \\
\left|C_{0}(t, x)-C_{0}(t, y)\right| \leqslant L_{3}|x-y|, \forall x, y \in \mathbb{R} .
\end{array}
$$

It is worth noting that we have introduced the impulsive effect term in Eq. (4.4) since the aggregate income may have a sudden change in some special cases if an economic crisis happens.

Substituting (4.2) and (4.3) in (4.4), we obtain

$$
\begin{cases}y^{\Delta}=\delta\left[C_{0}(t, y)+c y^{\sigma}+I_{0}(t, y)+G(t, y)-y\right], & t \in\left(\mathscr{R}_{\mathbb{T}}(\tau, \varepsilon)\right) \backslash \cup \mathfrak{B}^{\varepsilon}, t \neq t_{k_{i}, \varepsilon}, i \in \mathbb{Z}, \\ \Delta y\left(t_{k_{i}, \varepsilon}\right)=I\left(y\left(t_{k_{i}, \varepsilon}\right)\right)=y\left(t_{k_{i}, \varepsilon}^{+}\right)-y\left(t_{k_{i}, \varepsilon}^{-}\right), & t=t_{k_{i}, \varepsilon} \in \mathfrak{B}^{\varepsilon}, i \in \mathbb{Z} .\end{cases}
$$

Now, using the formula $y^{\sigma}=y+\mu y^{\Delta}$ and assuming that $1-\delta c \mu \neq 0$ for $t>a$, we have

$$
\left\{\begin{array}{c}
y^{\Delta}=\frac{\delta(c-1)}{1-\delta c \mu(t)} y+\frac{\delta\left(C_{0}(t, y)+I_{0}(t, y)+G(t, y)\right)}{1-\delta c \mu(t)}:=a(t) y+g(t, y), \\
t \in\left(\mathscr{R}_{\mathbb{T}}(\tau, \varepsilon)\right) \backslash \cup \mathfrak{B}^{\varepsilon}, t \neq t_{k_{i}, \varepsilon}, i \in \mathbb{Z}, \\
\Delta y\left(t_{k_{i}, \varepsilon}\right)=I\left(y\left(t_{k_{i}, \varepsilon}\right)\right)=y\left(t_{k_{i}, \varepsilon}^{+}\right)-y\left(t_{k_{i}, \varepsilon}^{-}\right), t=t_{k_{i}, \varepsilon} \in \cup \mathfrak{B}^{\varepsilon}, i \in \mathbb{Z} .
\end{array}\right.
$$


Note that $\mathbb{T}$ is an almost-complete closedness time scale, thus the graininess functions $\left|\mu_{\varepsilon}(t)-\mu(t)\right|<\varepsilon$.

Moreover, we assume that $\left|I\left(y_{1}\right)-I\left(y_{2}\right)\right| \leqslant L\left|y_{1}-y_{2}\right|$, for all $y_{1}, y_{2} \in \mathbb{R}$, and $c<1$ or $\mu(t)>\frac{1}{c \delta}$ for every $t \in \mathbb{T}$, i.e,

$$
c<1 \text { or } \mu_{\varepsilon}(t)>\frac{1}{c \delta}-\varepsilon \text { for every } t \in \mathscr{R}_{\mathbb{T}}(\tau, \varepsilon),
$$

and then we find that (4.5) admits exponential dichotomy with the projection $P=E$, where $E$ is the identity projection, i.e., $\|T(t, s)\|=\left|X(t) X^{-1}(s)\right| \leqslant K_{0} e_{\ominus \omega}(t, s), t \geqslant s, t, s \in \mathscr{R}_{\mathbb{T}}(\tau, \varepsilon)$. Hence, $\left(A_{1}\right)-\left(A_{2}\right)$ of Theorem 3.16 are satisfied. Further, if we assume that

$$
\max _{1 \leqslant i \leqslant 3}\left\{L_{i}\right\}<\frac{\omega}{2 K_{0}(1+(\bar{\mu}+\varepsilon) \omega)} \text { and } L<\frac{1-e_{\ominus \omega}\left(\theta_{\varepsilon}, 0\right)}{2 K_{0}},
$$

where $\theta_{\varepsilon}=\inf _{i} t_{k_{i}, \varepsilon}^{1}$ and $\left\{t_{k_{i}, \varepsilon}\right\} \in \mathfrak{B}^{\varepsilon}$, then $\left(A_{3}\right)$ of Theorem 3.16 is also satisfied. Therefore, we conclude that Eq. (4.5) has a weighted piecewise pseudo double-almost periodic solution. Furthermore, if $\max \left\{\mathrm{L}_{1}, \mathrm{~L}_{2}, \mathrm{~L}_{3}\right\}$ is small enough, we can easily see that all the conditions of Theorem 3.17 are also satisfied. Hence, Eq. (4.5) has a unique weighted piecewise pseudo double-almost periodic solution which is exponentially stable.

Application 4.3. Consider the following dynamic equation on an almost-complete closedness time scale $\mathbb{T}$ :

$$
\left\{\begin{array}{l}
x^{\Delta}(t)=A x(t)+f(t, x), \quad t \in\left(\mathscr{R}_{\mathbb{T}}(\tau, \varepsilon)\right) \backslash \cup \mathfrak{B}^{\varepsilon}, t \neq t_{k_{i}, \varepsilon}=k_{i}, k_{i} \in I_{0}, \\
\Delta x\left(t_{k_{i}, \varepsilon}\right)=I_{k}\left(t_{k_{i}, \varepsilon}, x\right), \quad t=t_{k_{i}, \varepsilon}=k_{0} \in \cup \mathfrak{B}^{\varepsilon},
\end{array}\right.
$$

where

$$
\begin{aligned}
\mathbb{T} & =\mathbb{P}_{a,|\sin \sqrt{3} t+\sin \sqrt{7} t|}, k_{i} \in I_{0}:=\left\{k_{0} \in\left(\mathscr{R}_{\mathbb{T}}(\tau, \varepsilon)\right) \cap\left(\cup_{k=-\infty}^{+\infty}\{k+|\sin \sqrt{3} k+\sin \sqrt{7} k|\}\right), k \in \mathbb{Z}\right\}, \\
A & =\left(\begin{array}{cc}
-7 & 0 \\
0 & -7
\end{array}\right), f(t, x)=\left(\begin{array}{c}
\frac{1}{30} \cos \sqrt{2} t x_{2} \\
\frac{1}{40} \sin \sqrt{3} t x_{1}
\end{array}\right), t \neq t_{k_{i}, \varepsilon}=k_{i}, i \in \mathbb{Z}, \\
I_{k}\left(t_{k_{i}, \varepsilon}, x\right) & =\left(\begin{array}{c}
\frac{3}{40} \hat{d} \cos 2 \sqrt{3} k_{i} x_{1}\left(k_{i}\right) \\
\frac{5}{60} \hat{d} \sin 2 \sqrt{2} k_{i} x_{2}\left(k_{i}\right)
\end{array}\right), t=t_{k_{i}, \varepsilon}=k_{i}, i \in \mathbb{Z} .
\end{aligned}
$$

Assume that for all $t \in \mathbb{T}$, there exists some $\varepsilon_{0}>0$ such that $\left|\mu(t)-\frac{1}{7}\right| \geqslant \varepsilon_{0}$. Hence, we can obtain

$$
\left|\mu_{\varepsilon}(t)-\frac{1}{7}\right| \geqslant|| \mu(t)-\mu_{\varepsilon}(t)|-| \mu(t)-\frac{1}{7}|| \geqslant \varepsilon_{0}-\varepsilon, t \in \mathscr{R}_{\mathbb{T}}(\tau, \varepsilon),
$$

which implies that $\mu_{\varepsilon}(t) \neq \frac{1}{7}$ for $\varepsilon<\varepsilon_{0}$.

Therefore, for all $t \in \mathscr{R}_{\mathbb{T}}(\tau, \varepsilon), E+\mu(t) A$ is invertible, so $A \in \mathcal{R}$. It is a routine calculation to show that the eigenvalues of the coefficient matrix $A$ are $\lambda_{1}=\lambda_{2}=-7$, and hence in view of Theorem 5.35 in [7, pp.201], the P-matrices are given by

$$
P_{0}=\left(\begin{array}{ll}
1 & 0 \\
0 & 1
\end{array}\right), P_{1}=\left(A-\lambda_{1} E\right) P_{0}=\left(\begin{array}{ll}
0 & 0 \\
0 & 0
\end{array}\right)
$$

We choose $r_{1}^{\Delta}=-7 r_{1}, r_{1}\left(t_{0}\right)=1, r_{2}^{\Delta}=r_{1}-7 r_{2}, r_{2}\left(t_{0}\right)=0$. Solving the first IVP for $r_{1}$, we find $r_{1}=$ $e_{-7}\left(t, t_{0}\right)$. Next, solving the second IVP, that is, $r_{2}^{\Delta}=-7 r_{2}+e_{-7}\left(t, t_{0}\right), r_{2}\left(t_{0}\right)=0$, we obtain

$$
r_{2}=e_{-7}\left(t, t_{0}\right) \int_{t_{0}}^{t} \frac{\Delta s}{1-7 \mu(s)}
$$


Thus, from Theorem 5.35 in [7, pp.201], we get

$$
e_{A}\left(t, t_{0}\right)=r_{1}(t) P_{0}+r_{2}(t) P_{1}=e_{-7}\left(t, t_{0}\right)\left(\begin{array}{ll}
1 & 0 \\
0 & 1
\end{array}\right)
$$

Therefore, for $t>s$, we have

$$
\left\|X(t) P_{0} X^{-1}(s)\right\|=\left\|e_{-7}\left(t, t_{0}\right)\left(\begin{array}{ll}
1 & 0 \\
0 & 1
\end{array}\right) e_{\ominus-7}\left(s, t_{0}\right)\left(\begin{array}{ll}
1 & 0 \\
0 & 1
\end{array}\right)\right\| \leqslant \sqrt{2} e_{\ominus 3}(t, s) .
$$

Hence, Eq. (4.6) admits an exponential dichotomy with a projection $P=E$, where $E$ is the identity projection. Further, since $L_{1}=\frac{\sqrt{2}}{30}, L_{2}=\frac{3 \sqrt{3}}{20} \hat{d}, K_{0}=\sqrt{2}, \omega=3$, for $0 \leqslant \bar{\mu} \leqslant 2$ and $\hat{d}<1-e_{\ominus \omega}\left(\theta_{\varepsilon}, 0\right)$, we have

$$
\frac{\mathrm{K}_{0} \mathrm{~L}_{1}(1+(\bar{\mu}+\varepsilon) \omega)}{\omega}+\frac{\mathrm{K}_{0} \mathrm{~L}_{2}}{1-e_{\ominus \omega}\left(\theta_{\varepsilon}, 0\right)}<\frac{1}{45}(7+3 \varepsilon)<0.2222<1 \text { for } \varepsilon<1,
$$

and $(\ominus \omega) \oplus p=-\frac{\omega}{1+\mu_{\varepsilon} \omega}+K_{0} L_{1}(1+(\bar{\mu}+\varepsilon) \omega)-\frac{K_{0} L_{1} \omega \mu_{\varepsilon}(1+(\bar{\mu}+\varepsilon) \omega)}{\left(1+\mu_{\varepsilon} \omega\right)} \leqslant-0.2333<0$ for $\varepsilon<1$, where $p=K_{0} L_{1}(1+(\bar{\mu}+\varepsilon) \omega)$. Hence, by Theorems 3.16 and 3.17, Eq. (4.6) has a unique weighted pseudo double-almost periodic solution satisfying:

$$
x(t)=\int_{-\infty}^{t} e_{-7}(t, \sigma(s))\left(\begin{array}{ll}
1 & 0 \\
0 & 1
\end{array}\right)\left(\begin{array}{c}
\frac{1}{30} \cos \sqrt{2} s x_{2} \\
\frac{1}{40} \sin \sqrt{3} s x_{1}
\end{array}\right) \Delta s+\sum_{-\infty<k_{i}<t} e_{-7}\left(t, k_{i}\right)\left(\begin{array}{c}
\frac{3}{40} \cos 2 \sqrt{3} k_{i} x_{1}\left(k_{i}\right) \\
\frac{5}{60} \sin 2 \sqrt{2} k_{i} x_{2}\left(k_{i}\right)
\end{array}\right), i \in \mathbb{Z},
$$

which is exponentially stable.

Application 4.4. Consider the following impulsive BAM neural networks:

$$
\begin{cases}x_{i}^{\Delta}(t)=\sum_{j=1}^{m} a_{i j}(t) x_{j}(t)+\sum_{j=1}^{m} \alpha_{i j}(t) f_{j}\left(y_{j}(t)\right)+\gamma_{i}(t), & t \neq t_{k_{\xi}, \varepsilon}, t \in \mathscr{R}_{\mathbb{T}}(\tau, \varepsilon), \xi \in \mathbb{Z}, \\ \Delta x_{i}\left(t_{k_{\xi}, \varepsilon}\right)=a_{i k} x_{i}\left(t_{k_{\xi}, \varepsilon}\right)+I_{i k}\left(x_{i}\left(t_{k_{\xi}, \varepsilon}\right)\right)+p_{i k}, & t=t_{k_{\xi}, \varepsilon} \in \cup \mathfrak{B}^{\varepsilon}, i=1,2, \ldots, n, \\ y_{j}^{\Delta}(t)=\sum_{i=1}^{n} b_{j i}(t) y_{j}(t)+\sum_{i=1}^{n} \beta_{j i}(t) g_{i}\left(x_{i}(t)\right)+v_{j}(t), t \neq t_{k_{\xi}, \varepsilon}, & t \in \mathscr{R}_{\mathbb{T}}(\tau, \varepsilon), \xi \in \mathbb{Z}, \\ \Delta y_{j}\left(t_{k_{\xi}, \varepsilon}\right)=b_{j k} y_{j}\left(t_{k_{\xi}, \varepsilon}\right)+J_{j k}\left(y_{j}\left(t_{k_{\xi}, \varepsilon}\right)\right)+w_{j k}, & t=t_{k_{\xi}, \varepsilon} \in \cup \mathfrak{B}^{\varepsilon}, j=1,2, \ldots, m,\end{cases}
$$

where $n, m$ are the numbers of neurons in layers, $x_{i}(t)$ and $y_{j}(t)$ denote the activations of the ith neuron and the $j$ th neuron at time $t, a_{i j}$ and $b_{j i}$ represent the rate with which the $i$ th neuron and the $j$ th neuron will reset their potential to the resting state in isolation when they are disconnected from the network and the external inputs at time $t, f_{j}, g_{i}$ are the input-output functions (the activation functions), $\alpha_{i j}, \beta_{j i}$ are elements of feedback templates at time $t, \gamma_{i}, v_{j}$ denote biases of the $i$ th neuron and the $j$ th neuron at time $t, \Delta x_{i}\left(t_{k_{\xi}, \varepsilon}\right)=x_{i}\left(t_{k_{\xi}, \varepsilon}^{+}\right)-x_{i}\left(t_{k_{\xi}, \varepsilon}^{-}\right), \Delta y_{j}\left(t_{k_{\xi}, \varepsilon}\right)=y_{j}\left(t_{k_{\xi}, \varepsilon}^{+}\right)-y_{j}\left(t_{k_{\varepsilon}, \varepsilon}^{-}\right)$are impulses at moments $t_{k_{\xi}, \varepsilon}$ and $t_{k_{1}}<t_{k_{2}}<\ldots$ is a strictly increasing sequence such that $\lim _{\xi \rightarrow \infty} t_{k_{\xi}, \varepsilon}=+\infty, a_{i k}, b_{j k}, p_{i k}, w_{j k} \in \mathbb{R}, I_{i k}, J_{j k} \in$ $C(\mathbb{R}, \mathbb{R}), i=1,2, \ldots, n, j=1,2, \ldots, m$.

We introduce the following conditions.

$\left(H_{1}\right)$ The functions $a_{i j}, b_{j i}, \alpha_{i j}, \beta_{j i}, \gamma_{i}, v_{j} \in P C_{r d}(\mathbb{T}, \mathbb{R})$ are weighted piecewise pseudo double-almost periodic on $\mathbb{T}, i=1,2, \ldots, n, j=1,2, \ldots, m$. We denote by $\lambda_{i}(t)$ the eigenvalues of matrix $A(t)$. There exist positive constants $\overline{\alpha_{i j}}, \overline{\beta_{j i}}, \lambda$ such that

$$
\sup _{t \in \mathbb{T}}\left|\alpha_{i j}\right|=\overline{\alpha_{i j}}<\infty, \sup _{t \in \mathbb{T}}\left|\beta_{j i}\right|=\overline{\beta_{j i}}<\infty, \operatorname{Re} \lambda_{i_{0}}(t)<-\lambda, i_{0}=1,2, \ldots, m+n .
$$


$\left(H_{2}\right)\left\{a_{i k}\right\},\left\{b_{j k}\right\},\left\{p_{i k}\right\},\left\{w_{j k}\right\}$ are weighted pseudo almost periodic sequences and $I_{i k}, J_{j k}$ are weighted pseudo almost periodic uniformly with respect to $x_{i}, y_{j} \in \mathbb{R}$, respectively, satisfying $I_{i k}(0)=$ $I_{j k}(0)=0, i=1,2, \ldots, n, j=1,2, \ldots, m$, and the following holds

$$
\left|I_{i k}(x)-I_{i k}(y)\right| \leqslant I_{i}|x-y|,\left|J_{j k}(x)-J_{j k}(y)\right| \leqslant J_{j}|x-y|, x, y \in \mathbb{R}, k \in \mathbb{Z} .
$$

$\left(H_{3}\right)$ The functions $f_{j}, g_{i} \in C(\mathbb{R}, \mathbb{R}), i=1,2, \ldots, n, j=1,2, \ldots, m$, satisfy

$$
0<\sup _{x \in \mathbb{R}}\left|f_{j}(x)\right|<\infty, f_{j}(0)=0,0<\sup _{x \in \mathbb{R}}\left|g_{i}(x)\right|<\infty, g_{i}(0)=0,
$$

and there exist constants $F_{j}>0$ and $G_{i}>0$ such that

$$
\left|f_{j}(x)-f_{j}(y)\right|<F_{j}|x-y|, \quad\left|g_{i}(x)-g_{i}(y)\right|<G_{i}|x-y|, x, y \in \mathbb{R} .
$$

$\left(\mathrm{H}_{4}\right)$ The set of sequences $\left\{\mathrm{t}_{\mathrm{k}_{\varepsilon}, \varepsilon}^{j}\right\}$ are equipotentially almost periodic and $\inf _{\xi} t_{k_{\varepsilon, \varepsilon}}^{1}=\theta_{\varepsilon}>0$, where $t_{k_{\xi}, \varepsilon}^{j}=t_{k_{\xi}, j}-t_{k_{\xi}, \varepsilon}, \xi \in \mathbb{Z}, j \in \mathbb{Z}$.

$\left(\mathrm{H}_{5}\right) \mathrm{r}<1$, where

$$
r=\frac{K(1+\lambda(\bar{\mu}+\varepsilon))}{\lambda}\left(\sum_{i=1}^{n} \sum_{j=1}^{m}\left(\overline{\alpha_{i j}} F_{j}+\overline{\beta_{j i}} G_{i}\right)\right)+\frac{K}{1-e_{-\lambda}\left(\theta_{\varepsilon}, 0\right)}\left(\sum_{i=1}^{n} I_{i}+\sum_{j=1}^{m} J_{j}\right),
$$

where $\sup _{\xi} e_{-\lambda}\left(t_{k_{\xi+1}, \varepsilon}, t_{k_{\xi}, \varepsilon}\right):=e_{-\lambda}\left(\theta_{\varepsilon}, 0\right)$.

$\left(\mathrm{H}_{6}\right)(\ominus \lambda) \oplus \mathrm{p}<0$, where

$$
p=K(1+(\bar{\mu}+\varepsilon) \lambda) \sum_{i=1}^{n} \sum_{j=1}^{m}\left(\overline{\alpha_{i j}} F_{j}+\overline{\beta_{j i}} G_{i}\right) .
$$

From the hypotheses $\left(\mathrm{H}_{1}\right)-\left(\mathrm{H}_{6}\right)$, it follows that all conditions of Theorems 3.16 and 3.17 are satisfied, and thus the system (4.7) has a unique weighted piecewise pseudo double-almost periodic solution which is exponentially stable.

\section{Acknowledgment}

This work was done while Chao Wang was a visiting scholar at Texas A\&M University-Kingsville. This work is supported by Youth Fund of NSFC (No. 11601470), Tian Yuan Fund of NSFC (No. 11526181), Yunnan Province Science and Technology Department Applied Basic Research Project of China (No. 2014FB102) and Dong Lu Youth Excellent Teachers Development Program of Yunnan University (No. wx069051).

\section{References}

[1] R. P. Agarwal, M. Bohner, Basic calculus on time scales and some of its applications, Results Math., 35 (1999), 3-22. 1

[2] R. P. Agarwal, M. Bohner, D. O’Regan, A. Peterson, Dynamic equations on time scales: a survey, Dynamic equations on time scales, J. Comput. Appl. Math., 141 (2002), 1-26. 1

[3] R. P. Agarwal, D. O'Regan, Some comments and notes on almost periodic functions and changing-periodic time scales, Electr. J. Math. Anal. Appl., 6 (2018), 125-136. 1, 2.7

[4] M. U. Akhmet, M. Turan, The differential equations on time scales through impulsive differential equations, Nonlinear Anal., 65 (2006), 2043-2060. 1

[5] S. Bochner, Beiträge zur Theorie der fastperiodischen Funktionen, (German) Math. Ann., 96 (1927), 119-147. 1

[6] M. Bohner, G. S. Guseinov, Double integral calculus of variations on time scales, Comput. Math. Appl., 54 (2007), 45-57. 3 
[7] M. Bohner, A. Peterson, Dynamic equations on time scales, An introduction with applications, Birkhäuser Boston, Inc., Boston, MA, (2001). 1, 2, 2.1, 2.2, 2.3, 4.3

[8] T. Caraballo, D. Cheban, Almost periodic and almost automorphic solutions of linear differential/difference equations without Favard's separation condition, I, J. Differential Equations, 246 (2009), 108-128. 1

[9] T. Caraballo, D. Cheban, Almost periodic and almost automorphic solutions of linear differential/difference equations without Favard's separation condition, II, J. Differential Equations, 246 (2009), 1164-1186. 1

[10] C. Cuevas, E. Hernández, M. Rabelo, The existence of solutions for impulsive neutral functional differential equations, Comput. Math. Appl., 58 (2009), 744-757. 1

[11] C. Cuevas, A. Sepúlveda, H. Soto, Almost periodic and pseudo-almost periodic solutions to fractional differential and integro-differential equations, Appl. Math. Comput., 218 (2011), 1735-1745. 1

[12] T. Diagana, Weighted pseudo almost periodic functions and applications, C. R. Math. Acad. Sci. Paris, 343 (2006), 643-646. 1

[13] T. Diagana, Existence of weighted pseudo-almost periodic solutions to some classes of nonautonomous partial evolution equations, Nonlinear Anal., 74 (2011), 600-615. 1

[14] A. M. Fink, Almost periodic differential equations, Lecture Notes in Mathematics, Springer-Verlag, Berlin-New York, (1974). 1, 2

[15] J. Gao, Q.-R. Wang, L.-W. Zhang, Existence and stability of almost-periodic solutions for cellular neural networks with time-varying delays in leakage terms on time scales, Appl. Math. Comput., 237 (2014), 639-649. 1

[16] E. Hernández, M. Rabello, H. R. Henríquez, Existence of solutions for impulsive partial neutral functional differential equations, J. Math. Anal. Appl., 331 (2007), 1135-1158. 1

[17] S. Hilger, Ein Maßkettenkalkäul mit anwendung auf zentrumsmannigfaltigkeiten, Ph.D thesis, Universität Wäurzburg, (1988). 1

[18] S. Hilger, Analysis on measure chains-a unified approach to continuous and discrete calculus, Results Math., 18 (1990), 18-56. 1

[19] B. Jackson, Partial dynamic equations on time scales, J. Comput. Appl. Math., 186 (2006), 391-415. 4.1

[20] E. R. Kaufmann, Y. N. Raffoul, Periodic solutions for a neutral nonlinear dynamical equation on a time scale, J. Math. Anal. Appl., 319 (2006), 315-325. 1

[21] J. Liang, J. Zhang, T.-J. Xiao, Composition of pseudo almost automorphic and asymptotically almost automorphic functions, J. Math. Anal. Appl., 340 (2008), 1493-1499. 1

[22] C. Pötzsche, Exponential dichotomies for dynamic equations on measure chains, Proceedings of the Third World Congress of Nonlinear Analysts, Part 2, Catania, (2000), Nonlinear Anal., 47 (2001), 873-884. 3

[23] A. M. Samoǐlenko, N. A. Perestyuk, Impulsive differential equations, With a preface by Yu. A. Mitropolskiřand a supplement by S. I. Trofimchuk, Translated from the Russian by Y. Chapovsky, World Scientific Series on Nonlinear Science, Series A: Monographs and Treatises, World Scientific Publishing Co., Inc., River Edge, NJ, (1995). 1

[24] G. T. Stamov, Almost periodic solutions of impulsive differential equations, Lecture Notes in Mathematics, Springer, Heidelberg, (2012). 1

[25] I. Stamova, Stability analysis of impulsive functional differential equations, De Gruyter Expositions in Mathematics, Walter de Gruyter GmbH \& Co. KG, Berlin, (2009). 1

[26] C. C. Tisdell, A. Zaidi, Basic qualitative and quantitative results for solutions to nonlinear, dynamic equations on time scales with an application to economic modelling, Nonlinear Anal., 68 (2008), 3504-3524. 4.2, 4.2

[27] C. Wang, Almost periodic solutions of impulsive BAM neural networks with variable delays on time scales, Commun. Nonlinear Sci. Numer. Simul., 19 (2014), 2828-2842. 1

[28] C. Wang, R. P. Agarwal, A further study of almost periodic time scales with some notes and applications, Abstr. Appl. Anal., 2014 (2014), 11 pages. 1, 2

[29] C. Wang, R. P. Agarwal, Relatively dense sets, corrected uniformly almost periodic functions on time scales, and generalizations, Adv. Difference Equ., 2015 (2015), 9 pages. 1, 2.4

[30] C. Wang, R. P. Agarwal, A classification of time scales and analysis of the general delays on time scales with applications, Math. Methods Appl. Sci., 39 (2016), 1568-1590. 1, 2, 2.7, 2.12, 3.5

[31] C. Wang, R. P. Agarwal, Almost periodic dynamics for impulsive delay neural networks of a general type on almost periodic time scales, Commun. Nonlinear Sci. Numer. Simul., 36 (2016), 238-251. 1, 2, 2.7, 3.5

[32] C. Wang, R. P. Agarwal, D. O'Regan, Compactness criteria and new impulsive functional dynamic equations on time scales, Adv. Difference Equ., 2016 (2016), 41 pages. 2

[33] C. Wang, R. P. Agarwal, D. O'Regan, Periodicity, almost periodicity for time scales and related functions, Nonauton. Dyn. Syst., 3 (2016), 24-41. 1, 2.7, 2.10, 2.12, 3.5

[34] C. Wang, R. P. Agarwal, D. O'Regan, Piecewise double-almost periodic functions on almost-complete closedness time scales with generalizations, submitted. 3

[35] C. Y. Zhang, Pseudo-almost-periodic solutions of some differential equations, J. Math. Anal. Appl., 181 (1994), 62-76. 1

[36] C. Y. Zhang, Pseudo almost periodic solutions of some differential equations, II, J. Math. Anal. Appl., 192 (1995), $543-561$. 1 